\title{
WestVirginiaUniversity
}

THE RESEARCH REPOSITORY @ WVU

Graduate Theses, Dissertations, and Problem Reports

2005

\section{The hyperspace graph of connected subgraphs}

Likin C. Simon Romero

West Virginia University

Follow this and additional works at: https://researchrepository.wvu.edu/etd

\section{Recommended Citation}

Simon Romero, Likin C., "The hyperspace graph of connected subgraphs" (2005). Graduate Theses, Dissertations, and Problem Reports. 2321.

https://researchrepository.wvu.edu/etd/2321

This Dissertation is protected by copyright and/or related rights. It has been brought to you by the The Research Repository @ WVU with permission from the rights-holder(s). You are free to use this Dissertation in any way that is permitted by the copyright and related rights legislation that applies to your use. For other uses you must obtain permission from the rights-holder(s) directly, unless additional rights are indicated by a Creative Commons license in the record and/ or on the work itself. This Dissertation has been accepted for inclusion in WVU Graduate Theses, Dissertations, and Problem Reports collection by an authorized administrator of The Research Repository @ WVU.

For more information, please contact researchrepository@mail.wvu.edu. 


\section{The Hyperspace Graph of Connected Subgraphs}

\section{Likin C. Simon Romero}

Dissertation submitted to the

Eberly College of Arts and Sciences

in partial fulfillment of the requirements

for the degree of

Doctor of Philosophy

in

Mathematics

Sam B. Nadler, Jr., Ph.D., Chair

Henry W. Gould, M.A.

Hong Jian Lai, Ph. D.

Eddie Fuller, Ph. D.

Patricia Pellicer Covarruvias, Ph.D.

Leonardo Golubovic, Ph.D.

Department of Mathematics

Morgantown, West Virginia

2005

Keywords: Hyperspace Graph, Size Levels, Hyperspaces

Copyright 2005 Likin C. Simon Romero 


\title{
ABSTRACT \\ THE HYPERSPACE GRAPH OF CONNECTED SUBGRAPHS
}

\author{
Likin C. Simon Romero
}

Given a connected graph $G$, the hyperspace graph of connected subgraphs $\mathcal{C}(G)$ is defined. The graph $\mathcal{C}(G)$ is such that every vertex represents a connected subgraph of $G$. It is shown that every connected graph $G$ has a unique graph $\mathcal{C}(G)$. A characterization of a path, a cycle and the 3 -star by their corresponding hyperspace graphs of connected subgraphs is shown. An special geometric representation $\mathcal{R}(G)$ of $\mathcal{C}(G)$ in an euclidean space is presented. A set $\mathcal{P}(G)$ is constructed based on $\mathcal{R}(G)$. When $G$ is a topological tree, $\mathcal{P}(G)$ and the hyperspace of subcontinua of $G$ are homeomorphic.

Given a graph $G$, the size of $G$ is the cardinality of the edge set. A special kind of subgraphs of $\mathcal{C}(G)$ is studied; given a non-negative integer $n$, the $n$-th size level of $G$, denoted by $\mathcal{Q}_{n}(G)$ is defined. This graph is the induced graph in $\mathcal{C}(G)$ of all the connected subgraphs of $G$ with size $n$. Relations between $G$, the graphs $\mathcal{Q}_{n}(G)$ and $\mathcal{C}(G)$ are analyzed. 
To Nicole,

for her love and support. 


\section{Contents}

List of Figures $\quad$ vi

1 INTRODUCTION 1

2 PRELIMINARIES $\quad 6$

2.1 DEFINITIONS AND NOTATION $\ldots \ldots \ldots \ldots \ldots \ldots$

2.2 CONNECTED GRAPHS . . . . . . . . . . . . . . . . . . 10

2.3 REMOVABLE AND COMPLEMENTARY EDGES. . . . . . . . . . . . . 11

3 SIZE LEVELS 16

3.1 DEFINITION AND BASIC PROPERTIES. . . . . . . . . . . . . . . . 17

3.2 THE CORE AND THE JOIN. . . . . . . . . . . . . . . . . . . 20

3.3 SIZE LEVELS OF PATHS AND CYCLES. . . . . . . . . . . . . . . . . 24

3.4 AN IMPORTANT SIZE LEVEL. . . . . . . . . . . . . . . . . . . 30

3.5 DEGREES IN THE SIZE LEVELS. . . . . . . . . . . . . . . . . . 34

3.6 NEIGHBORHOODS AND CUT VERTICES . . . . . . . . . . . . 36

3.7 HAMILTONIAN LEVELS . . . . . . . . . . . . . . . . . . . 46

4 THE GRAPH $\mathcal{C}(\mathbf{G}) \quad \mathbf{5 3}$

4.1 DEFINITION AND BASIC PROPERTIES . . . . . . . . . . . . . 53

$4.2 \quad$ PLANARITY OF $\mathcal{C}(\mathbf{G}) \ldots \ldots \ldots \ldots \ldots \ldots$

4.3 UNIQUENESS OF $\mathcal{C}(\mathbf{G}) \ldots \ldots \ldots \ldots \ldots$

5 RELATIONS BETWEEN $\mathcal{C}(\mathbf{G})$ AND C $(\mathbf{X}) \quad \mathbf{7 0}$

5.1 A PARTICULAR GEOMETRIC REPRESENTATION OF $\mathcal{C}(\mathbf{G}) \ldots \ldots . . .71$

$5.2 \quad$ INDUCED SET OF $\mathcal{C}(\mathbf{G}) \ldots \ldots \ldots \ldots \ldots \ldots \ldots$

5.3 RELATIONS BETWEEN $\mathcal{C}(\mathbf{G})$ AND $\mathbf{C}(\mathbf{X})$ FOR TREES. . . . . . . . . . 82

6 QUESTIONS $\quad 89$

6.1 QUESTIONS ABOUT THE SIZE LEVELS . . . . . . . . . . . . . . . . 89

6.2 QUESTIONS ABOUT $\mathcal{C}(\mathbf{G}) \ldots \ldots \ldots \ldots \ldots \ldots$

6.3 OTHER HYPERSPACES . . . . . . . . . . . . . . . . . . . . . 91

6.4 INFINITE GRAPHS AND DIGRAPHS . . . . . . . . . . . . . . . . 92 
Bibliography 


\section{List of Figures}

1.1 A model for $C(X)$ when $X$ is a simple triod. . . . . . . . . . . 2

2.1 Some operations with graphs. . . . . . . . . . . . . . . . . 9

3.1 Examples of size levels of some graphs. . . . . . . . . . . . . . . . . . 18

3.2 Graphs in the first case of Lemma $85 \ldots \ldots \ldots$. . . . . . . . . . . 42

4.1 Examples of the graph $\mathcal{C}(G) \ldots \ldots \ldots \ldots \ldots \ldots$

4.2 Proof of Lemma $117 \ldots \ldots \ldots \ldots$

5.1 The sets $R_{v}, S_{v}$ and $M_{v}$ when $v$ is a degree 3 vertex. . . . . . . 79

5.2 The induced set of the 3 -star $\ldots \ldots \ldots \ldots \ldots$. . . . . . . . 81 


\section{Acknowledgments}

I would like to thank first and foremost to my advisor Dr. Sam B. Nadler for his advise and patience. I want also to express my gratitude to all the members of my committee: Professor Hong Jian Lai, Professor Henry W. Gould, Professor Eddie Fuller, Professor Patricia Pellicer Covarruvias and Professor Leonardo Golubovic. Their disposition is greatly appreciated. I thank my teachers here in West Virginia University for sharing their knowledge with me. I would like to thank specially to Professors Harvey Diamond and Fred King for their help and support. To Raylene DeLeon, Meredith McCartle and Joann Mayhew, I want to say how much I will miss them, they made the life of everyone in the Mathematics Department easier and happier.

To my parents Cecilia and Gabriel, I want to thank their education, love and support. They made sure that I always have the necessary things to go after my dreams. Also, thanks to the rest of my family, although they are far away, they will be very close to me. I want to say to my friends in Mexico how much I miss them. I thank my friends and (hopefully) soon to be colleagues Benjamin Espinoza and Jorge Martinez, it was a pleasure to spend the time with them here in Morgantown. They were my home away from home. I would like to mention also Miguel, Luisa, Indira, Anish, Maria, Donna and all my friends here in the United States. We have a lot of unforgettable experiences together.

Finally, I would like to thank the love of my life and fiancee Nicole, being with her is the best thing that had happen to me. I am looking forward to spending the rest of my life with you. 


\section{Chapter 1}

\section{INTRODUCTION}

In topology, a hyperspace of a given topological space is a specific collection of subsets to which a special topology is given. In particular, if we consider a continuum $X$ (compact, connected, metric space), we can define the hyperspace of subcontinua $C(X)$ by the collection of all subcontinua together with a special topology. In Chapter 5, we mention the metric that induces the topology in $C(X)$ (Definition 153). In Figure 1.1 we show a model of the hyperspace $C(X)$ when the space $X$ is a simple triod. An excellent reference book is [5] written by A. Illanes and S. B. Nadler, Jr. Different questions are asked about the relations between the topological properties of $X$ and those of $C(X)$ and vice versa.

The main idea of our work is to define an analogous concept in graph theory. In [8], given a graph, S. B. Nadler, Jr. defines a new graph whose vertices represent subsets of vertices of the original graph. This concept generates the ideas for our work. Our objective is to define and analyze a natural analogue of the hyperspace $C(X)$ in the setting of graph theory. We start by extending an important concept in the study of topological hyperspaces. 


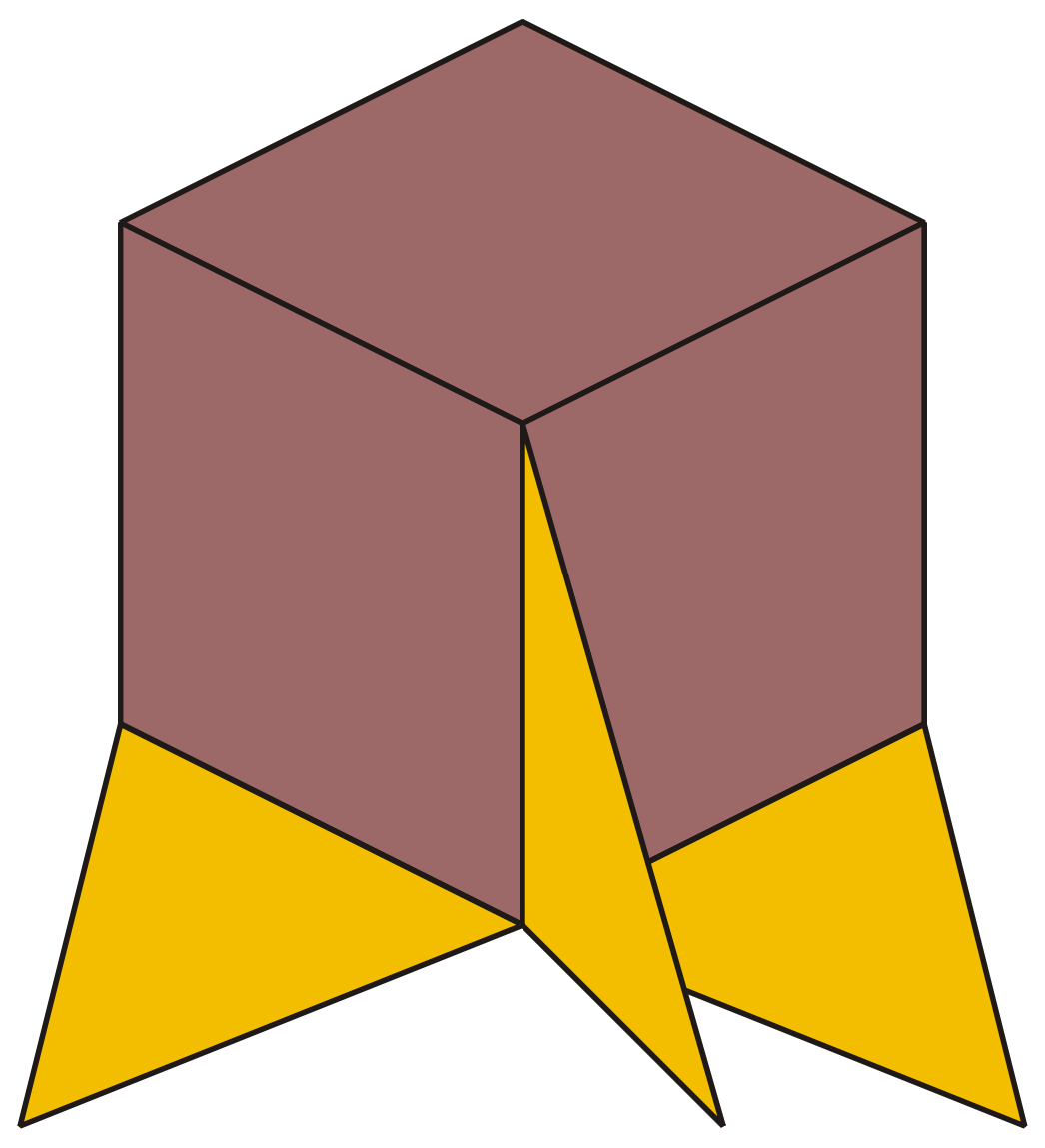

Figure 1.1: A model for $C(X)$ when $X$ is a simple triod. 
In $C(X)$, we can define a non negative real-valued function, called a Whitney function, that "measures" how big the subcontinua are. In graph theory, the analogous concept is size. The size of a graph is the cardinality of the edge set (Definition 3). A Whitney level is a continuum in which every point represents a subcontinuum with the same "measure". In other words, the inverse image of a non-negative number under a Whitney function. Whitney levels are an important tool for analyzing $X$ as well as $C(X)$. There are topological properties such that, if the space $X$ has the given property, then all nondegenerate Whitney levels of $X$ have that property. These properties are called Whitney properties. Being an arc and being a simple closed curve are Whitney properties. Having cutpoints is not a Whitney property. On the other hand, there are properties such that if all the positive Whitney levels of $X$ have such property, then the space $X$ has the property too. These properties are called Whitney reversible properties. It is known that being an arc and being a simple closed curve are Whitney reversible properties. An extensive study of these two kinds of properties appear in the book cited before ([5], Chapter VIII).

In our case, we start defining the $n$-th size level graph in Chapter 3 (Definition 36 ); this $n$-th size level graph is such that its vertices represent connected subgraphs with size $n$. These size level graphs are connected (Theorem 50). Naturally, we can ask which properties are such that, if the graph $G$ has the given property, then all the nondegenerate size levels of $G$ have that property. We call such properties size properties. We can also ask which properties are such that if all the nonzero nondegenerate size levels of $G$ have such property, then the graph $G$ has the property. We call these properties size reversible properties. In Section 3.3, we show being a path is a size property (Corollary 52) and being 
a cycle is a size property (Corollary 54). Moreover, we show that if one size level of $G$ is a path, then $G$ is a path (Theorem 63); thus, being a path is a size reversible property. For cycles, we show that if one size level of $G$ is a cycle with size greater than three, then $G$ is a cycle of of the same size (Theorem 64). This implies that being a cycle with size greater than three is a size reversible property.

As we mentioned, having cutpoints is not a Whitney property. Analogously, having cutvertices is not a size property. We study results about this level in Section 3.4. This leads us to question, what levels have cutvertices? In Section 3.6, we characterize the subgraphs that are cutvertices in their corresponding levels (Theorem 91).

One property that is studied in graph theory is the property of being hamiltonian. A hamiltonian graph is a graph $G$ such that there is a cycle in $G$ containing every vertex of $G$. So, we can ask if being hamiltonian is a size property or a size reversible property. This question was one of the first questions that we originally asked. In Section 3.7, we prove that all the nonzero nondegenerate size levels of stars are hamiltonian (Corollary 102). Thus, being hamiltonian is not size reversible.

In Chapter 4, we define the graph $\mathcal{C}(G)$ (Definition 104). The vertices of $\mathcal{C}(G)$ represent the connected subgraphs of $G$. Note that the size levels of $G$ are all subgraphs of $\mathcal{C}(G)$. One fundamental question to ask is, when is $\mathcal{C}(G)$ planar? In Section 4.2, we show that the paths, cycles and the 3 -star are the only types of graphs having planar hyperspace graphs of connected subgraphs (Theorem 118). In topological hyperspaces, the analogous result is that the only non-degenerate continua whose hyperspaces of subcontinua are planar are the arc and the simple closed curve. 
In topological hyperspaces, a continuum $X$ has unique hyperspace if, for every continuum $Y$ such that $C(X)$ is homeomorphic to $C(Y)$, we have that $X$ is homeomorphic to $Y$. It is shown in [2] that finite topological graphs, different from the arc and the simple closed curve, have unique hyperspace of subcontinua (the arc and the simple closed curve have a 2-cell as their hyperspace of subcontinua). In Section 4.3, we prove that if two graphs have isomorphic hyperspace graphs, then the graphs are isomorphic (Theorem 130). This includes paths and cycles, the counterparts of the arc and the simple closed curve in topological hyperspace theory.

A topological graph $X$ can be looked as an abstract graph $G$. It is natural to ask about the relations between $C(X)$ and $\mathcal{C}(G)$. We give a particular geometric representation of the hyperspace graph $\mathcal{C}(G)$ in a particular euclidean space (Chapter 5). This representation induces a set in that euclidean space; when $G$ is a tree, the set with the euclidean topology is homeomorphic to $C(X)$, where $X$ is the topological analogous to the graph $G$. 


\section{Chapter 2}

\section{PRELIMINARIES}

In this thesis we work with graphs. First, we define the concepts of basic graph

theory. In particular, we define the size in Definition 3, that is the number of edges of the graph. We also discuss some operations between graphs and we use these operations to construct new graphs. Since we want to define graphs whose vertex sets are connected subgraphs, we give the definition of connected graph in Section 2.2, Definition 17, together with some other definitions and remarks. All these concepts are explained deeply in [1] and $[4]$.

In Section 2.3, we define three new concepts, namely, removable edge, complementary edge set and complementary edge. In Lemma 32, we give some useful characterizations of some particular subgraphs. 


\subsection{DEFINITIONS AND NOTATION}

Definition 1 A graph $G$ is a finite non-empty set denoted by $V(G)$ together with a set (possibly empty) of unordered pairs of distinct elements of $V(G)$, denoted by $E(G)$. The set $V(G)$ is called the vertex set of $\mathbf{G}$ and the set $E(G)$ is called the edge set of $\mathbf{G}$. The elements of $V(G)$ are called vertices of $G$ and the pairs in $E(G)$ are called edges of $G$. If the edge e is determined by the pair $v$ and $w$, we say that e joins $v$ and $w$. If two vertices are joined by an edge, they are said to be adjacent.

Definition 2 A vertex is contained in an edge, or an edge is incident with (or has) a vertex, if the vertex is one of the members of the pair that defines the edge. An edge e is said to be incident with a graph $G$ if e is not an edge of $G$ but e is incident with a vertex of $G$. If an edge e is incident with $G$ in two vertices, e is called a handle of $G$.

Definition 3 The size of a graph $G$ is the cardinality of its edge set.

Definition 4 Let $G$ be a graph and let $v$ be a vertex of $G$. The degree of $\mathbf{v}$ in $\mathbf{G}$ is the number of edges of $G$ incident with $v$. This number is denoted by $\operatorname{deg}_{G} v$.

Definition 5 If a graph $G$ is such that $V(G)=\{v\}$ and $E(G)=\varnothing$, then $G$ is said to be a degenerate graph and denoted by $G=\{v\}$. 
Definition 6 A graph $G$ is complete if every two vertices are adjacent. We denote by $\mathcal{K}_{n}$ the complete graph with $n$ vertices.

Definition 7 A graph $G$ is an $\mathbf{n}$-star if the size of $G$ is $n$ and there is a vertex $v$ such that every edge of $G$ is incident to $v$.

Definition 8 A graph $K$ is said to be a subgraph of a graph $G$ if $V(K)$ is a subset of $V(G)$ and $E(K)$ is a subset of $E(G)$. We denote $K$ being a subgraph of $G$ by writing $K \subset G$.

Definition 9 Let $G$ be a graph and $K$ be a subgraph of $G$. We refer by $\mathbf{E}(\mathbf{K}, \mathbf{G})$ to the set of all edges of $G$ incident with $K$. Note that $E(G, G)=\varnothing$.

Definition 10 If $\mathcal{E}$ is a non-empty subset of $E(G)$, the subgraph induced by $\mathcal{E}$, denoted by $\langle\mathcal{E}\rangle$, is the graph whose vertex set consists of those vertices of $G$ that are contained in at least one edge in $\mathcal{E}$ and whose edge set is exactly $\mathcal{E}$. In other words:

$V(\langle\mathcal{E}\rangle)=\{v \in V(G)$ : e is incident with $v$ for some e $\in E(G)\}$

$E(\langle\mathcal{E}\rangle)=\mathcal{E}$

We denote $\left\langle\mathrm{e}_{1}, \mathrm{e}_{2}, \ldots, \mathrm{e}_{n}\right\rangle$ instead of $\left\langle\left\{\mathrm{e}_{1}, \mathrm{e}_{2}, \ldots, \mathrm{e}_{n}\right\}\right\rangle$.

Note that if $\mathbf{e}=v w$, then $\langle\mathbf{e}\rangle$ is the graph with vertex set $\{v, w\}$ and edge set $\{\mathbf{e}\}$.

Definition 11 Let $K$ and $L$ be graphs. The union of $K$ and $L$ is the graph $G$ defined by $V(G)=V(K) \cup V(L)$ and $E(G)=E(K) \cup E(L)$. We write the union of $K$ and $L$ by $K \cup L$. Note that both $K$ and $L$ are subgraphs of $G$. 
We often consider the union of a graph $G$ and a single edge graph $\langle e\rangle$ so, in order to make some expressions shorter, we write $G \cup$ e instead of $G \cup\langle\mathrm{e}\rangle$.

Definition 12 Let $\mathcal{V}$ be a subset of $V(G)$. The graph $\mathbf{G}$ minus $\mathcal{V}($ denoted by $G-\mathcal{V})$ is defined by the following sets: the vertex set is $V(G)-\mathcal{V}$ and the edge set is

$$
E(G)-\{\mathrm{e} \in E(G) \text { : e contains at least one vertex in } \mathcal{V}\}
$$

We also consider frequently the graph $G-\{v\}$. Again, to simplify the expressions, we write $G-v$ instead of $G-\{v\}$.

Definition 13 If $G$ is a graph and $\mathcal{E}$ is a subset of $E(G)$, then we say that the graph $\mathbf{G}$ minus $\mathcal{E}$ (denoted by $G-\mathcal{E}$ ) is the graph defined by letting $V(G)$ be the vertex set and $E(G)-\mathcal{E}$ be the edge set of $G-\mathcal{E}$ respectively. If $\mathcal{E}=\{\mathrm{e}\}$, then we denote $G-\{\mathrm{e}\}$ by $G-\mathrm{e}$

In Figure 2.1, we give an example of a graph $G$ in (a), $G \cup$ e in (b), $G-v$ in (c), $G-\mathrm{f}$ in (d), $G-\{w, z\}$ in (e) and $G-\{\mathrm{f}, \mathrm{g}, \mathrm{h}\}$ in (f).

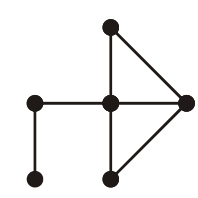

(a)



(b)

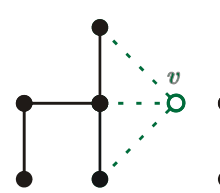

(c)

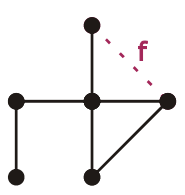

(d)

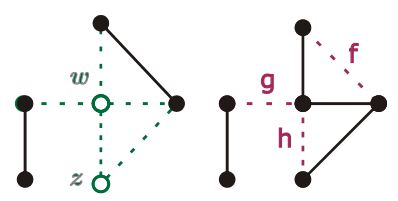

(e)

(f)

Figure 2.1: Some operations with graphs. 


\subsection{CONNECTED GRAPHS}

Definition $14 A$ walk in a graph $G$ is a finite sequence of at least two vertices $\mathcal{W}$, namely $\mathcal{W}=v_{0} v_{1} v_{2} \ldots v_{n}$, such that $v_{i}$ and $v_{i+1}$ are adjacent in $G$ for all $i=0,1,2, \ldots, n-1 . \quad A$ walk is a closed walk if the first vertex and the last vertex of the sequence are the same. In other words, $v_{0}=v_{n}$. The length of a walk $\mathcal{W}$ is just the corresponding index $n$ of the sequence $\mathcal{W}$. If the length is zero, the walk has only one vertex and it is called a trivial (or degenerate) walk. Two vertices are consecutive in the walk if they are consecutive elements in the sequence.

Definition 15 A path is a walk in which every two vertices of the walk are different.

Definition 16 A cycle is a closed walk such that $v_{i}=v_{j}$ if and only if $\{i, j\}=\{0, n\}$.

Definition 17 A graph is said to be connected if for every two vertices of the graph, there is a path joining those two vertices. A graph is disconnected if the graph is not connected.

It is easy to check that the previous definition is equivalent to saying that every vertex in the graph can be joined to a fixed vertex of the same graph by a path.

Remark 18 If $K$ and $L$ are connected graphs such that $K$ and $L$ have a vertex in common, then $K \cup L$ is connected. Note also that if $K$ is a connected subgraph of a graph $G$ and $\mathrm{e}$ is an edge of $G$ that is incident with $K$, then $K \cup$ e is a connected subgraph of $G$.

Definition 19 A tree is a connected graph that contains no cycle. 
Definition 20 Let $G$ be a connected graph, let $v$ and $w$ be two vertices of $G$. The distance from $v$ to $w$, denoted by $d(v, w)$, is the minimum of the sizes of the paths joining $v$ and $w$ if $v \neq w$, or zero if $v=w$. The diameter of $G$ is the greatest distance between two vertices of $G$.

Definition 21 A separation of a graph $G$ is a pair of vertex disjoint subgraphs $I$ and $J$ such that $G=I \cup J$ and no vertex of $I$ is adjacent to a vertex of $J$.

Remark 22 Note that $G$ has a separation if and only if $G$ is disconnected.

Definition 23 A vertex $v$ of a connected graph $G$ is a cut-vertex if $G-\{v\}$ is not connected.

\subsection{REMOVABLE AND COMPLEMENTARY EDGES.}

Definition 24 A vertex $v$ of $G$ is an interior vertex if $\operatorname{deg}_{G}(v) \geq 2$. On the other hand, a vertex $v$ of $G$ is a endvertex if $\operatorname{deg}_{G}(v)=1$. An edge e of $G$ is a terminal edge if e contains an endvertex.

Definition 25 An edge e of $G$ is a cycle edge if there is a cycle in $G$ containing e as an edge.

Definition 26 An edge e of $G$ is removable if e is either a cycle or a terminal edge. 
Remark 27 Let $G$ be a connected graph and let e be a cycle edge of $G$. Then $K=G-\mathrm{e}$ is connected. Moreover $V(K)=V(G)$ and $E(K)=E(G)-\{\mathrm{e}\}$.

Lemma 28 Let $G$ be a connected graph, let e be a terminal edge of $G$ and let $v$ be the endvertex of $G$ contained in e. Then $K=G-\{v\}$ is connected. Moreover, $V(K)=$ $V(G)-\{v\}$ and $E(K)=E(G)-\{\mathrm{e}\}$.

Proof. Suppose that $K$ is not connected and let e be the terminal edge incident with $v$. Then, there are $I$ and $J$ subgraphs of $K$ such that $I$ and $J$ form a separation of $K$. So, e can be incident to either $I$ or $J$ but not to both. Without loss of generality, assume that e is incident with $I$. It is clear that $I \cup$ e and $J$ form a separation of $G$. This contradicts the connectedness of $G$. Therefore, $K$ is a connected subgraph of $G$.

Definition 29 Let $G$ be a graph and let $K$ be a subgraph of $G$. The set $E(G)-E(K)$ is called the complementary edge set of $K$ in $G$ and it is denoted by $\mathcal{E}_{K, G}$. In particular, if $E(G)-E(K)=\{\mathrm{e}\}$, then $\mathrm{e}$ is the complementary edge of $K$ in $G$ and it is denoted by $\mathrm{e}_{K, G}$.

Lemma 30 Let $G$ be a connected graph, let $K$ and $L$ be nondegenerate connected subgraphs of $G$ and let $\mathcal{E}_{K}$ and $\mathcal{E}_{L}$ be the complementary edge sets of $K$ and $L$ in $G$ respectively. Then $\mathcal{E}_{L} \subset \mathcal{E}_{K}$ if and only if $K \subset L$.

Proof. Assume that $\mathcal{E}_{L} \subset \mathcal{E}_{K}$. Hence, $\varnothing \neq E(K) \subset E(L)$, but since $K$ and $L$ are connected, then $V(K) \subset V(L)$. So, $K \subset L$. 
On the other hand, if $K \subset L$, we have that $E(K) \subset E(L)$. Thus, $\mathcal{E}_{L}=E(G)-$ $E(L) \subset E(G)-E(K)=\mathcal{E}_{L}$.

Remark 31 The previous lemma implies the following equivalence: if $K$ and $L$ are nondegenerate connected subgraphs of a connected graph $G$, then $\mathcal{E}_{K}=\mathcal{E}_{L}$ if and only if $K=L$. Also, $E(K)=E(L)$ if and only if $K=L$.

The next lemma is the characterization of the connected subgraphs with respect to their complementary edge. This characterization is one of the main tools that we use in later chapters. Recall the definition of size in Definition 3.

Lemma 32 Let $G$ be a connected graph of size $N$. An edge e is removable if and only if there is a unique connected subgraph $K$ of $G$ with size $N-1$ such that e is the complementary edge of $K$ in $G$. Moreover, if e is a terminal edge of $G$ with endvertex $v$, then $K=G-\{v\}$ and if e is a cycle edge of $G$, then $K=G-\mathrm{e}$.

Proof. If e is a removable edge, then e is either terminal or a cycle edge of $G$.

Assume e is a cycle edge of $G$. Define $K=G-\mathrm{e}$. Note that $K$ is a connected subgraph of $G$ with complementary edge e (Remark 27). If there is a connected subgraph $K^{\prime}$ of $G$ with size $N-1$ and not containing e as an edge, then $K=K^{\prime}$ (Remark 31).

In the case when e is a terminal edge, let $v$ be the corresponding endvertex of e in $G$. Let $K=G-\{v\}$. By Lemma $28, K$ is a connected subgraph of $G$ with complementary edge e and with size $N-1$. Uniqueness of $K$ comes from Remark 31 .

On the other hand, $K$ is obviously a connected subgraph of $G$ and e is the complementary edge of $K$ in $G$. Therefore, e is a removable edge. 
Lemma 33 Let $G$ be a connected graph with size $N \geq 1$. For every vertex $v$ in $G$, there is a connected subgraph $K$ with size $N-1$ such that $v$ is a vertex of $K$.

Proof. If $G$ contains a cycle edge, the result follows immediately from Remark 27. So, assume that $G$ is a tree.

Then $G$ has at least two endvertices. Let $w$ be an endvertex distinct from $v$ and let e be the corresponding terminal edge for $w$. By Lemma 32, there is a connected subgraph $K$ of $G$ with complementary edge set e. By Lemma 28, $V(K)=V(G)-\{w\}$. Then $v$ is a vertex of $K$.

Lemma 34 Let $G$ be a connected graph of size $n+1$. If $K$ and $L$ are two distinct nondegenerate subgraphs of $G$ with size $n$, then

$$
|E(K)-E(L)|=|E(L)-E(K)|=1 .
$$

Proof. Note that the following hold:

$$
\begin{aligned}
& |E(K)-E(L)|+|E(K) \cap E(L)|=|E(K)|=n \\
& |E(K)-E(L)|+|E(K) \cap E(L)|+|E(L)-E(K)| \leq|E(G)|=n+1
\end{aligned}
$$

It follows that $|E(L)-E(K)| \leq 1$. Note that $|E(L)-E(K)| \neq 0$ by the fact that that $K$ and $L$ have the same size and by the previous lemma. Therefore, $|E(L)-E(K)|=1$. A similar argument shows that $|E(K)-E(L)|=1$.

Lemma 35 Let $G$ be a connected graph and let $K$ be a connected subgraph of $G$ with size $n$. If there is an edge e incident with $K$, then there exists a connected subgraph $L$ of $G$ with size $n+1$ such that $K$ is a connected subgraph of $L$ with complementary edge e in $L$. Moreover, $L=K \cup \mathrm{e}$. 
Proof. Just define $L=K \cup$ e. Then $L$ is a connected graph containing $K$ and having size $n+1$. 


\section{Chapter 3}

\section{SIZE LEVELS}

Given a graph $G$ of size $N$ and a non-negative number $n$ less than or equal to $N$, we define a graph called the $n$-th size level of $G$. This graph has the property that every vertex represents a connected subgraph with size $n$. The idea of the size level graph was inspired by the concept of Whitney level (see Chapter 1).

In Section 3.1, we give the definition of the size level graph (Definition 36). The definition of adjacency in the size levels leads us immediately to Section 3.2. We define the concept of the core of two graphs (Definition 41) and we prove the uniqueness of the core (Theorem 42). We also define the join of two graphs, show the existence and uniqueness of the join (Theorem 46) and prove that the size levels are connected (Theorem 50).

We analyze the size levels of paths and cycles in Section 3.3. We also give a characterization of paths and cycles by their size levels (Theorems 51 and 53).

A very important size level (Section 3.4) is the one whose vertices are connected graphs that contain all but one of the edges of our original graph. This level has a very 
peculiar structure for graphs that are not big cycles (Theorem 72).

The degree of vertices of the size levels is discussed in Section 3.5. In Corollary 74, we give a formula for the degree. In Section 3.6, we show a characterization of cut-vertices in the size levels (Theorem 91). Finally, in Section 3.7, we show that the size levels of stars are hamiltonian.

\subsection{DEFINITION AND BASIC PROPERTIES.}

Definition 36 Let $G$ be a connected graph with size $N$. The $n$-th size level of $G$, denoted by $\mathbf{Q}_{n}(\mathbf{G})$, is the graph defined by follows:

- The vertex set of $\mathcal{Q}_{n}(G)$ is the set of all connected subgraphs of size $n$. In other words: $V\left(\mathcal{Q}_{n}(G)\right)=\{H$ is a connected subgraph of $G$ and $H$ has size $n\}$.

- If $n=0,\{v\}$ is adjacent to $\{w\}$ in $\mathcal{Q}_{0}(G)$ provided that $v$ is adjacent to $w$ in $G$.

- If $1 \leq n \leq N$, then $K$ is adjacent to $L$ in $\mathcal{Q}_{n}(G)$ provided that $K$ and $L$ are different and there is a connected subgraph $C$ of $G$ with size $n-1$ such that $C \subset K$ and $C \subset L$.

If two subgraphs $K$ and $L$ in the same size level are adjacent, we say that $K$ and $L$ are level adjacent in $\mathcal{Q}_{n}(G)$ (or $n$-level adjacent).

In Figure 3.1 we show the size levels of: (a) a path with size 3, (b) a cycle with size 4, (c) a 3-star and (d) the graph isomorphic to the letter $H$. 



(a)
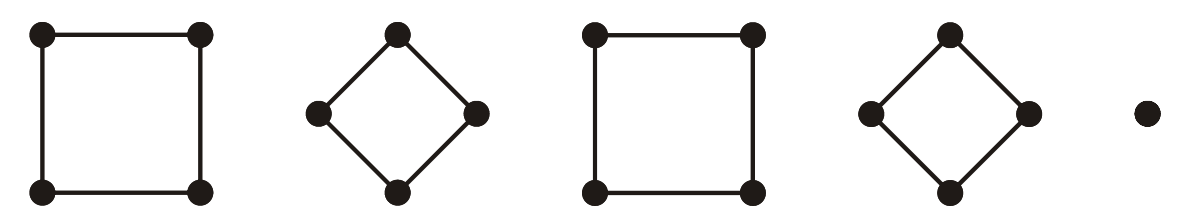

(b)


-

(c)



(d)
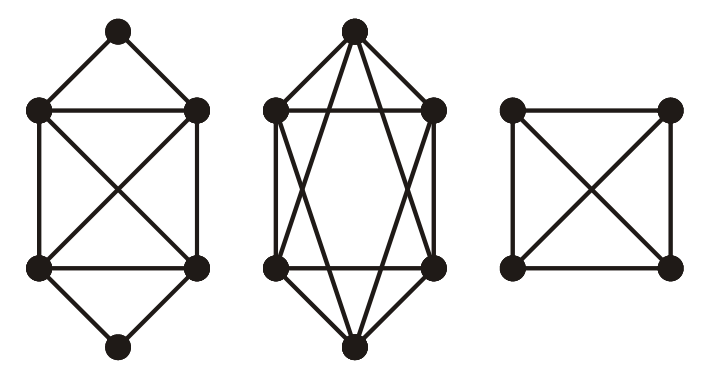

Figure 3.1: Examples of size levels of some graphs. 
Remark 37 Let $G$ be a connected graph with size $N$. Then

(1) $\mathcal{Q}_{N}(G)$ is a degenerate graph. This size level is called the degenerate size level.

(2) $\mathcal{Q}_{0}(G)$ is isomorphic to $G$. This size level is called the zero size level.

(3) $\mathcal{Q}_{1}(G)$ is just the line graph of $G$ (see [1] p.108).

Remark 38 Let $G$ be a connected graph and let $K$ be a connected subgraph of $G$ with size $n$. Then for every $m \leq n$ we have that $\mathcal{Q}_{m}(K)$ is a subgraph of $\mathcal{Q}_{m}(G)$.

Inspired by the Whitney properties and Whitney reversible properties, we can define the following:

Definition 39 A property of graphs is called a size property if, given a graph $G$ with the property, then all nondegenerate size levels of $G$ have the property. A property of graphs is called a size reversible property if whenever all the nonzero and nondegenerate size levels of a given graph $G$ have the property, then the graph $G$ itself has the property.

Note that, in Figure 3.1 (c), we can see that being a cycle is not a size reversible property.

The following lemma is a characterization of the subgraphs that are level adjacent to a given graph. 
Lemma 40 Let $K$ be a connected subgraph of $G$ of size $n \geq 1$ and let e be an edge of $G$ incident with $K$. Then there exists a connected subgraph $L$ of $G$ such that $K$ and $L$ are level adjacent and e is an edge of $L$.

Proof. Let $v$ be a vertex in common between e and $K$. Using Lemma 33 there is a connected subgraph $C$ of $K$ with size $n-1$ such that $v$ is a vertex of $C$. So, e is incident with $C$. Thus applying Lemma 35, there is a connected subgraph $L$ of $G$ with size $n$ such that $L=C \cup$ e. Since $C \subset K$ and $C \subset L, H$ and $K$ are level adjacent.

\subsection{THE CORE AND THE JOIN.}

Given the definition of adjacency for the size levels of a graph $G$, there are two important subgraphs of $G$ related to the edges of the size levels. Given a size level $\mathcal{Q}_{n}(G)$ and two level adjacent subgraphs $K$ and $L$ in $\mathcal{Q}_{n}(G)$, the common subgraph with size $n-1$ contained in $K$ and $L$ is called the core of $H$ and $K$. Given a size level $\mathcal{Q}_{n}(G)$ and two level adjacent subgraphs $K$ and $L$ in $\mathcal{Q}_{n}(G)$, the subgraph with size $n+1$ that contains both $K$ and $L$ is called the join of $K$ and $L$. These graphs help us to relate properties of consecutive levels.

Definition 41 Let $G$ be a connected graph and let $K$ and $L$ be $n$-level adjacent nondegenerate subgraphs of $G$ The graph $C$ is a core of $K$ and $L$, denoted by core $(K, L)$, if $C$ is a common connected subgraph $C$ of $K$ and $L$ with size $n-1$.

Theorem 42 Let $G$ be a connected graph. If $K$ and $L$ are two level adjacent subgraphs of $G$ with size $n \geq 1$, then there is a unique core of $K$ and $L$. 
Proof. Let $C$ be a core of $K$ and $L$. Suppose that there is a connected subgraph $C^{\prime}$ of both in $K$ and $L$ and with size $n-1$. Since $C$ and $C^{\prime}$ are subgraphs of $K$, we have that $\left|E\left(C^{\prime}\right)-E(C)\right|=1$ (by Lemma 34). Let e $\in E\left(C^{\prime}\right)-E(C)$. Note that e $\in$ $E(K) \cap E(L)$, so $E(C) \cup\{\mathrm{e}\} \subset E(K) \cap E(L) \subset K$ and since $|E(C) \cup\{\mathrm{e}\}|=n=|E(K)|$, then $|E(K) \cap E(L)|=n$. But this implies that $E(K)=E(L)$ and contradicts the fact that $K$ and $L$ are different.

Denote by $\operatorname{core}(K, L)$ the unique core of $K$ and $L$. Note that two different pairs of level adjacent subgraphs may have the same core.

Proposition 43 If $K$ and $L$ are two level adjacent subgraphs of $G$ with size $n+1$, then $\operatorname{core}(K, L)=\langle E(K) \cap E(L)\rangle$.

Proof. Let $C=\operatorname{core}(K, L)$ and let $\mathrm{e}_{K}$ and $\mathrm{e}_{L}$ be the complementary edges of $C$ in $K$ and in $L$ respectively. Then $E(K)=E(C) \cup\left\{\mathbf{e}_{K}\right\}$ and $E(L)=E(C) \cup\left\{\mathbf{e}_{L}\right\}$. This implies that $E(K) \cap E(L)=E(C)$ and since every vertex of $C$ is in an edge of $C$, it follows that $V(\langle E(K) \cap E(L)\rangle)=V(C)$. Therefore, $\operatorname{core}(K, L)=\langle E(K) \cap E(L)\rangle$.

Note that since $\operatorname{core}(K, L)$ is a subgraph of $K$ and $L$ we can talk about the complementary edge of $\operatorname{core}(K, L)$ in $K$ and in $L$. Here we have two remarks concerning the core and complementary edges:

Remark 44 Let $G$ be a connected graph, let $K$ and $L$ be level adjacent subgraphs of $G$ and let $C=\operatorname{core}(K, L)$. If $\mathrm{e}_{C, K}$ is the complementary edge of $C$ in $K$, then $K=C \cup \mathrm{e}_{C, K}$.

Remark 45 Let $G$ be a connected graph, let $K$ and $L$ be level adjacent subgraphs of $G$ and 
let $C=\operatorname{core}(K, L)$. If $\mathrm{e}_{C, K}$ is the complementary edge of $C$ in $K$, then $\mathrm{e}_{C, K}$ is incident with $L$.

The following theorem leads to a descriptive name for the other important subgraph related to two level adjacent subgraphs.

Theorem 46 Let $K$ and $L$ be two level adjacent subgraphs of $G$ with size $n$. Then there is a unique connected subgraph $U$ of $G$ with size $n+1$ such that $K$ and $L$ are both connected subgraphs of $U$. Moreover, if $K$ and $L$ are nondegenerate, then $U=K \cup L$.

Proof. If $n=0$, then $K$ and $L$ are degenerate. Let $K=\{v\}$ and $L=\{w\}$ where $v$ and $w$ are two distinct vertices of $G$. Since $K$ and $L$ are level adjacent, then $v$ and $w$ are adjacent in $G$. Let $\mathrm{e}=v w$ and let $U=\langle\mathrm{e}\rangle$. So, $K$ and $L$ are connected subgraphs of $U$. Since there is only one edge joining $v$ and $w$ in $G$, then $U$ has to be unique.

If $K$ and $L$ are nondegenerate, then let $U=K \cup L$. By Remark 18, we have that $U$ is a connected graph. It follows from Lemma 34 that the size of $U$ is $n+1$. If there is a connected subgraph $U^{\prime}$ of $G$ such that $K$ and $L$ are both subgraphs of $U^{\prime}$, then $U=U^{\prime}$ or $U$ and $U^{\prime}$ are level adjacent. If $U$ and $U^{\prime}$ are level adjacent, then by Theorem $42, K=L$. This is a contradiction to the fact that $K$ and $L$ are level adjacent. Therefore, $U=U^{\prime}$.

Definition 47 Let $G$ be a connected graph and let $K$ and $L$ be level adjacent subgraphs of $G$ with size $n$. The unique connected subgraph of $G$ with size $n+1$ and containing $K$ and $L$ is called the join of $K$ and $L$ and is denoted by join $(K, L)$. 
Here is how the core and the join are related.

Remark 48 Let $G$ be a connected graph, let $K$ and $L$ be level adjacent subgraphs of $G$, let $C=\operatorname{core}(K, L)$ and let $U=j \operatorname{join}(K, L)$. If $\mathrm{e}_{C, K}$ is the complementary edge of $C$ in $K$ and $\mathbf{e}_{C, L}$ is the complementary edge of $C$ in $L$, then $U=\operatorname{core}(K, L) \cup\left\{\mathbf{e}_{C, K}, \mathbf{e}_{C, L}\right\}$. Moreover, $\mathrm{e}_{C, K}$ is the complementary edge of $L$ in $U$ and $\mathrm{e}_{C, L}$ is the complementary edge of $K$ in $U$.

Lemma 49 Let $H, K$ and $L$ be three connected subgraphs of $G$ such that $H$ and $K$ are level adjacent and $K$ and $L$ are also level adjacent. If $U=j \operatorname{join}(H, K), V=j \operatorname{oin}(K, L)$ and $U \neq V$, then $U$ and $V$ are level adjacent.

Proof. As a consequence of the definition of the join, we have that $K \subset U$ and $K \subset V$, therefore $U$ and $V$ are level adjacent.

Theorem 50 All size levels of a connected graph are connected.

Proof. Let $G$ be a connected graph of size $N$. The proof is done by induction on $n$.

Since $\mathcal{Q}_{0}=G, \mathcal{Q}_{0}$ is connected.

Assume that $\mathcal{Q}_{n}$ is connected for some $n<N$.

Suppose that $\mathcal{Q}_{n+1}$ is not connected. So, there is a separation $\mathcal{M}$ and $\mathcal{N}$ of $\mathcal{Q}_{n+1}$. Let $\mathcal{O}$ be the subgraph of $\mathcal{Q}_{n}$ induced by the vertex set:

$V(\mathcal{O})=\left\{K \in V\left(\mathcal{Q}_{n}\right): K\right.$ is a subgraph of $L$ for some $\left.L \in V(\mathcal{M})\right\}$.

Also, define $\mathcal{P}$ by the subgraph of $\mathcal{Q}_{n}$ induced by the vertex set: 
$V(\mathcal{P})=\left\{K \in V\left(\mathcal{Q}_{n}\right): K\right.$ is a subgraph of $L$ for some $\left.L \in V(\mathcal{N})\right\}$.

For every $K \in V\left(\mathcal{Q}_{n}\right)$, since $n<N$, there is an edge e incident with $K$. Hence, $K \subset K \cup$ e. However, $\mathcal{M}$ and $\mathcal{N}$ is a separation of $\mathcal{Q}_{n+1}$, then $K \cup \mathrm{e} \in V(\mathcal{M}) \cup V(\mathcal{N})$. This implies that $V\left(\mathcal{Q}_{n}\right)=V(\mathcal{O}) \cup V(\mathcal{P})$.

Since $\mathcal{Q}_{n}$ is connected, there is an edge in $\mathcal{Q}_{n}$ joining a vertex $L$ in $\mathcal{O}$ with a vertex $L^{\prime}$ in $\mathcal{P}$.

Let $U=$ join $\left(L, L^{\prime}\right)$. Without loss of generality, assume that $U \in V(\mathcal{M})$ (the argument for the other case is practically the same).

So, in particular, $L^{\prime} \subset U$. On the other hand, since $L^{\prime} \in V(\mathcal{P})$, there is $U^{\prime} \in V(\mathcal{N})$ such that $L^{\prime} \subset U^{\prime}$. By definition $U$ and $U^{\prime}$ are level adjacent. That is a contradiction since $U$ and $U^{\prime}$ are in different sides of the separation.

\subsection{SIZE LEVELS OF PATHS AND CYCLES.}

In the hyperspace of subcontinua, the Whitney levels of an arc are arcs and the Whitney levels of a simple closed curve are simple closed curves (see [5], 27.3 and 27.4). It seems natural to ask if the analogous statement is true for paths and cycles. The answer is yes (Theorems 51 and 53). Actually, we characterize paths and cycles with size greater than 3 by their size levels (Theorems 63 and 64 ).

Theorem 51 Every nondegenerate size level of a path is a path.

Proof. If the path has size one, then the only nondegenerate size level is the zero level, which is a path. Hence, we consider a path of length greater than one. 
Let $P=v_{0} v_{1} \ldots v_{N}$ be a path and let $\mathcal{Q}_{n}$ be a nondegenerate size level of $P$.

Consider the path $O=v_{0} v_{1} \ldots v_{N-n}$ and the function $\phi: V\left(\mathcal{Q}_{n}\right) \longrightarrow V(O)$ that assigns to each $K_{i}=v_{i} v_{i+1} \ldots v_{i+n}$ the endvertex $v_{i}$, i.e. $\phi\left(K_{i}\right)=v_{i}$.

This function is clearly one-to-one.

Let $K_{i}$ be level adjacent to $K_{j}$. Note that if $K_{i}$ and $K_{j}$ are degenerate, then $v_{i}$ and $v_{j}$ are adjacent. Assume that $K_{i}$ and $K_{j}$ are nondegenerate. Consider $J=\operatorname{core}\left(K_{i}, K_{j}\right)$. Since $J$ is a connected subgraph of a path, $J$ is a path.

If $J$ is degenerate, then $K_{i}$ and $K_{j}$ have size 2. Thus $J=\left\{v_{i}\right\}$ or $J=\left\{v_{i+1}\right\}$. Therefore, $v_{j}=v_{i}$ and $v_{j}=v_{i+1}$.

Assume, $J$ is a nondegenerate subpath of $K_{i}$ we have that $J=v_{i+1} v_{i+2} \ldots v_{i+n}$ or $J=v_{i} v_{i+1} \ldots v_{i+(n-1)}$.

If $J=v_{i+1} v_{i+2} \ldots v_{i+n}$, then $K_{j}=v_{i+1} v_{i+2} \ldots v_{i+n} v_{i+n+1}$, since $K_{i} \neq K_{j}$. Therefore, $v_{j}=v_{i+1}$

If $J=v_{i} v_{i+1} \ldots v_{i+(n-1)}$, then $K_{j}=v_{i-1} v_{i} v_{i+1} \ldots v_{i+(n-1)}$, since $K_{i} \neq K_{j}$. Therefore, $v_{j}=v_{i-1}$

This implies that $\phi$ is an isomorphism.

Corollary 52 Being a path is a size property.

Theorem 53 Every nondegenerate size level of a cycle of length $N$ is also a cycle of length $N$

Proof. Let $C=v_{0} v_{1} \ldots v_{N-1} v_{0}$ be a cycle and let $\mathcal{Q}_{n}$ be a nondegenerate size level of $C$. 
Consider the function $\phi: V\left(\mathcal{Q}_{n}\right) \longrightarrow V(C)$ that assigns to each $K_{i}=v_{i} v_{i+1} \ldots v_{i+n}$ the endvertex $v_{i}$, i.e. $\phi\left(K_{i}\right)=v_{i}$. All our indices are considered modulus $N$.

This function is clearly one-to-one.

Let $K_{i}$ be level adjacent to $K_{j}$. Note that if $K_{i}$ and $K_{j}$ are degenerate, then $v_{i}$ and $v_{j}$ are adjacent. Assume that $K_{i}$ and $K_{j}$ are nondegenerate. Consider $J=\operatorname{core}\left(K_{i}, K_{j}\right)$. Since $J$ is a proper connected subgraph of a cycle, $J$ is a path.

If $J$ is degenerate, then $K_{i}$ and $K_{j}$ have size 2. Thus $J=\left\{v_{i}\right\}$ or $J=\left\{v_{i+1}\right\}$. Therefore, $v_{j}=v_{i}$ and $v_{j}=v_{i+1}$.

Assume, $J$ is a nondegenerate subpath of $K_{i}$, we have that $J=v_{i+1} v_{i+2} \ldots v_{i+n}$ or $J=v_{i} v_{i+1} \ldots v_{i+(n-1)}$.

If $J=v_{i+1} v_{i+2} \ldots v_{i+n}$, then $K_{j}=v_{i+1} v_{i+2} \ldots v_{i+n} v_{i+n+1}$, since $K_{i} \neq K_{j}$. Therefore, $v_{j}=v_{i+1}$

If $J=v_{i} v_{i+1} \ldots v_{i+(n-1)}$, then $K_{j}=v_{i-1} v_{i} v_{i+1} \ldots v_{i+(n-1)}$, since $K_{i} \neq K_{j}$. Therefore, $v_{j}=v_{i-1}$

This implies that $\phi$ is an isomorphism.

Corollary 54 Being a cycle is a size property.

Corollary 55 If a connected graph contains a cycle of length $N$, then its $n$-th size level for $n<N$ contains a cycle of length $N$.

Proof. Just apply Theorem 53.

Next, we give a characterization of the graphs that have a level that is a path and the graphs that have a level that is a cycle. First, we need some lemmas. 
Lemma 56 Let $G$ be a connected graph and let $K$ be a proper connected subgraph of $G$ with size $n$. If $K$ contains a cycle, then $\operatorname{deg}_{\mathcal{Q}_{n}(G)} K \geq 3$.

Proof. Let $\mathrm{e}_{1}, \mathrm{e}_{2}$ and $\mathrm{e}_{3}$ be three cycle edges of $K$ and let $\mathrm{f}$ be an edge incident with $K$. Then $K-\mathrm{e}_{1}, K-\mathrm{e}_{2}$ and $K-\mathrm{e}_{3}$ are connected. Thus, the graphs $\left(K-\mathrm{e}_{1}\right) \cup \mathrm{f}$, $\left(K-\mathrm{e}_{2}\right) \cup \mathrm{f}$ and $\left(K-\mathrm{e}_{3}\right) \cup \mathrm{f}$ are level adjacent to $K$. Therefore, $\operatorname{deg}_{\mathcal{Q}_{n}(G)} K \geq 3$.

Lemma 57 Let $G$ be a connected graph. If $K$ is a connected subgraph of $G$ with size $n \geq 2$, if $v$ is an endvertex of $K$ and if there is an edge e that is a handle of $K$ not having $v$ as a vertex, then there exists a connected subgraph $L$ of $G$, level adjacent to $K$ such that $\operatorname{deg}_{\mathcal{Q}_{n}(G)} L \geq 3$

Proof. Let $\mathrm{f}$ be the terminal edge of $K$ containing $v$. Using Lemma 28, we have that $K-\{v\}$ is connected and e is incident with $K-\{v\}$ in two vertices $u$ and $w$. Define $L=(K-\{v\}) \cup e$. Since $K-\{v\}$ is connected, there is a path $P$ in $K-\{v\}$ joining $u$ and $w$. Then $P \cup$ e is a cycle of $L$. Using Lemma 56, we have that $\operatorname{deg}_{\mathcal{Q}_{n}(G)} L \geq 3$.

Lemma 58 Let $G$ be a connected graph and let $\mathcal{Q}_{n}$ be the $n$-th size level of $G$ with $n \geq 3$. If all the vertices of $\mathcal{Q}_{n}$ are paths in $G$, then $G$ is either a cycle or a path.

Proof. Assume there is a vertex $v$ of $G$ such that $\operatorname{deg}_{G}(v) \geq 3$. Hence, there are edges $\mathrm{e}_{1}, \mathrm{e}_{2}$ and $\mathrm{e}_{3}$ of $G$ incident with $v$. Let $T=\left\langle\mathrm{e}_{1}, \mathrm{e}_{2}, \mathrm{e}_{3}\right\rangle$. Note that $T$ is a connected subgraph of $G$ with size 3. Consider a subgraph $K$ of $G$ with size $n$ that contains $T$ as a subgraph. So, $K$ is not a path. This contradicts our hypothesis. 
Then every vertex of $G$ is of degree at most 2 . Therefore, $G$ has to be a cycle or a path.

Theorem 59 If the $n$-th size level of a graph is a path, then the graph itself is a path.

Proof. Let $G$ be a graph and let $\mathcal{Q}_{n}$ be the $n$-th size level of $G$ that is a path.

Assume first that $0<n<3$ and that there is a vertex $v$ of $G$ such that $\operatorname{deg}_{G}(v) \geq 3$.

Hence, there are edges $\mathrm{e}_{1}, \mathrm{e}_{2}$ and $\mathrm{e}_{3}$ of $G$ incident with $v$. Let $T=\left\langle\mathrm{e}_{1}, \mathrm{e}_{2}, \mathrm{e}_{3}\right\rangle$. Note that $T$ is a connected subgraph of $G$ with size 3 . So, $\mathcal{Q}_{n}(T)$ is a 3 -cycle that is a subgraph of $\mathcal{Q}_{n}$ (see Figure 3.1). This contradicts the fact that $\mathcal{Q}_{n}$ is a path. So, if $n<3$, then $G$ is either a cycle or a path. But by Theorem $53, G$ cannot be a cycle. Therefore, $G$ has to be a path.

We can assume that $n \geq 3$. Let $K$ be a subgraph of $G$ with size $n$. Since $\mathcal{Q}_{n}$ is a path, we have that $\operatorname{deg}_{\mathcal{Q}_{n}}(K) \leq 2$ and by Lemma $56, K$ is a tree. We will show that $K$ has to be a path.

Assume $K$ is a tree that is not a path. Let $v_{1}, v_{2}$ and $v_{3}$ be three endvertices of $K$ with corresponding terminal edges $\mathrm{f}_{1}, \mathrm{f}_{2}$ and $\mathrm{f}_{3}$, respectively. Since $K$ is a proper subgraph of $G$, there is an edge e of $G$ such that e is incident with $K$.

By Lemma 57, we can assume that e is not a handle. Without loss of generality, suppose that $\mathrm{e}$ is not incident with $v_{1}$ and $v_{2}$. Then $K$ is level adjacent to both $\left(K-\left\{v_{1}\right\}\right) \cup \mathrm{e}$ and $\left(K-\left\{v_{2}\right\}\right) \cup$ e. But $\left(K-\left\{v_{1}\right\}\right) \cup$ e is also level adjacent to $\left(K-\left\{v_{2}\right\}\right) \cup$ e. This implies that $\left\langle K,\left(K-\left\{v_{1}\right\}\right) \cup \mathrm{e},\left(K-\left\{v_{2}\right\}\right) \cup \mathrm{e}\right\rangle$ is a cycle contained in $\mathcal{Q}_{n}$. This is a contradiction. Therefore, $K$ is a path. 
So, all the subgraphs of $G$ with size $n$ are paths if $n \geq 3$. By Lemma $58, G$ has to be a path or a cycle. Again using Theorem 53, $G$ cannot be a cycle. Therefore, $G$ has to be a path.

Corollary 60 Being a path is a size reversible property.

Proof. Use Theorem 59.

Theorem 61 Let $G$ be a connected graph with size $N$. If the $n$-th size level of $G$ is a nondegenerate cycle for $3 \leq n \leq N-2$, then the graph itself is a cycle.

Proof. Let $\mathcal{Q}_{n}$ be the $n$-th size level of $G(3 \leq n \leq N-2)$ that is a cycle. Let $K$ be any subgraph of $G$ with size $n$. Since $\mathcal{Q}_{n}$ is a cycle, we have that $\operatorname{deg}_{\mathcal{Q}_{n}} K=2$ and by Lemma $56, K$ is a tree. We will show again that $K$ has to be a path.

Assume $K$ is a tree that is not a path. Let $v_{1}, v_{2}$ and $v_{3}$ be three endvertices of $K$. Note that $K$ is a proper subgraph of $G$ and has size at most $N-2$. Then there are edges $\mathrm{e}_{1}$ and $\mathrm{e}_{2}$ such that $\mathrm{e}_{1}$ is incident with $K$ and $\mathrm{e}_{2}$ is incident with $K \cup \mathrm{e}_{1}$.

By Lemma 57, we can assume that $\mathrm{e}_{1}$ is not incident to a pair of endvertices of $K$. Without loss of generality, suppose that $\mathrm{e}_{1}$ is not incident with $v_{1}$ and $v_{2}$. This implies that $K-\left\{v_{1}, v_{2}\right\}$ is connected and $\mathrm{e}_{1}$ is incident with it.

If $\mathrm{e}_{2}$ is not incident with $K$, then consider $L=\left(K-\left\{v_{1}\right\}\right) \cup \mathrm{e}_{1}, H=\left(K-\left\{v_{2}\right\}\right) \cup \mathrm{e}_{1}$ and $J=\left(L-\left\{v_{2}\right\}\right) \cup \mathrm{e}_{2}$. But observe that $L$ is level adjacent to $H, J$ and $K$. This contradicts the hypothesis of $\mathcal{Q}_{n}$ being a cycle.

If $\mathrm{e}_{2}$ is incident with $K$, then using Lemma 57, we can assume that $\mathrm{e}_{2}$ is not incident to a pair of endvertices of $K$. In particular, $\mathrm{e}_{2}$ is not incident with both $v_{1}$ and $v_{2}$. 
Assume that $\mathrm{e}_{2}$ is not incident to $v_{2}$.

So, $L=\left(K-\left\{v_{1}\right\}\right) \cup \mathrm{e}_{1}, L^{\prime}=\left(K-\left\{v_{1}\right\}\right) \cup \mathrm{e}_{2}$ and $L^{\prime \prime}=\left(K-\left\{v_{2}\right\}\right) \cup \mathrm{e}_{2}$ are level adjacent to $K$. This is again, a contradiction.

As a consequence, $K$ is a path in $G$. But, we did this for any subgraph $K$ of $G$ with size $n$; hence, all the subgraphs of $G$ with size $n \geq 3$ are paths. Thus, by Lemma 58 , $G$ has to be a path or a cycle. However, by Theorem $51, G$ cannot be a path. Therefore, $G$ has to be a cycle.

Corollary 62 Being a cycle with size greater than 3 is a size reversible property.

Proof. By Theorem 61, $G$ is a cycle.

Combining the theorems in this section, we arrive to the following two results:

Theorem 63 A graph is a path if and only if it has a size level that is a path.

Theorem 64 A graph is a cycle of size $N$ if and only if it has an $n$-th size level that is a nondegenerate cycle with $3 \leq n \leq N-2$.

The condition $n \leq N-2$ in the Theorem 64 is necessary: the $(N-1)$-st size level of a tree with size $N$ and exactly three endvertices is a cycle of size 3 .

\subsection{AN IMPORTANT SIZE LEVEL.}

Given a connected graph $G$ with size $N$, one very useful level to work with is the largest nondegenerate level, in other words, the $(N-1)$-st size level. The main result, 
Theorem 72, will help later in Section 4.3, to show that two graphs that have isomorphic hyperspace graph are isomorphic.

Theorem 65 Let $G$ be a connected graph with size $N$, let $K$ be a connected nondegenerate subgraph of $G$ with size $N-1$ and let e be a terminal edge of $G$ such that e is the complementary edge of $K$ in $G$. Then for any connected subgraph $L$ of $G$ with size $N-1$ distinct from $K, K$ is level adjacent to $L$.

Proof. Let $L$ be a connected subgraph of $G$ with size $N-1$ distinct from $K$ and let $\mathrm{f}$ be the complementary edge of $L$ in $G$. Since $K \neq L$, by Remark 31 , e $\neq \mathrm{f}$ and $\mathrm{f}$ is a removable edge of $K$. Then by Lemma 32, there is a connected subgraph $H$ of $K$ with size $N-2$ and complementary edge $f$ of $H$ in $K$. Now, $H$ has complementary edge set $\{e, f\}$ in $G$ and $L$ has complementary edge set $\{\mathbf{f}\}$ in $G$. Hence, by Lemma $30, H$ is a subgraph of $L$ with size $N-2$. Therefore, $K$ and $L$ are level adjacent.

We note the following corollary:

Corollary 66 Let $G$ be a tree with $k$ endvertices and size $N$. Then the $(N-1)$-st size level of $G$ is a complete graph with $k$ vertices.

Theorem 67 Let $G$ be a connected graph with size $N$, let $K$ be a connected nondegenerate subgraph of $G$ with size $N-1$ and let e be an edge of a cycle $Y$ of $G$ such that e is the complementary edge of $K$ in $G$. Then for any connected subgraph $L$ of $G$ with size $N-1$ with complementary edge not in the cycle $Y, K$ and $L$ are level adjacent. 
Proof. Let $L$ be a connected subgraph of $G$ with size $N-1$ and let $\mathrm{f}$ be the complementary edge of $L$ in $G$ but not in the cycle $Y$. So, $Y$ is a cycle of $L$ and e is a cycle edge of $L$. This implies that the subgraph $C=L-\mathrm{e}$ is a connected subgraph of $L$ with size $N-2$. Now, the complementary edge set of $C$ in $G$ is $\{\mathrm{e}, \mathbf{f}\}$. By Lemma $30, C$ is a subgraph of $K$. Therefore, $K$ and $L$ are level adjacent.

Now, we analyze the structure of the last nondegenerate level. First, we need some definitions and a result.

Definition 68 A graph $B$ is a bipartite graph provided that there is a partition $V$ and $W$ of the vertex set of $B$ such that every edge of $B$ joins a vertex in $V$ with a vertex in $W$. A bipartite graph with a partition $V$ and $W$ is a complete bipartite graph if every vertex of $V$ and every vertex of $W$ are adjacent. We denote by $\mathcal{K}_{n, m}$ the complete bipartite graph such that $V$ has $n$ elements and $W$ has $m$ elements.

Definition 69 A subgraph $S$ of a graph $G$ is said to be spanning (or spans $G$ ) if $V(S)=$ $V(G)$

Definition 70 A graph $G$ is said to be stitched if $G$ contains a spanning complete bipartite subgraph.

Remark 71 As a consequence of the previous definitions, a graph $G$ is stitched if and only if there is a partition $V$ and $W$ of the vertex set of $G$ such that for every $v \in V$ and $w \in W$ we have that $v$ is adjacent to $w$. 
Theorem 72 Let $G$ be a connected graph with size $N$. Then $G$ is not a cycle of size greater than 4 if and only if $\mathcal{Q}_{N-1}(G)$ is stitched.

Proof. If $G$ is a cycle with size $N$, by Theorem 53 , we have that $\mathcal{Q}_{N-1}(G)$ is a cycle with size $N$. Then $\mathcal{Q}_{N-1}(G)$ can be spanned by $\mathcal{K}_{1,2}$ if $N=3$ or by $\mathcal{K}_{2,2}$ if $N=4$. Therefore, they are stitched.

If $G$ is a tree, by Corollary 66 , we have that $\mathcal{Q}_{N-1}(G)$ is a complete graph and therefore, $G$ is stitched.

So, we can assume that $G$ is neither a cycle nor a tree.

For any vertex $K$ of $\mathcal{Q}_{N-1}(G)$, define $\mathrm{e}_{K}$ to be the complementary edge of $K$ in $G$. By Lemma 32, we have that every vertex of $\mathcal{Q}_{N-1}(G)$ can be characterized by its complementary edge.

Consider the following sets of vertices of $\mathcal{Q}_{N-1}(G)$.

Since $G$ is not a tree, $G$ contains a cycle $C_{0}$; let:

$$
W=\left\{K \in V\left(\mathcal{Q}_{N-1}(G)\right): \mathrm{e}_{K} \in E\left(C_{0}\right)\right\}
$$

If $G$ has terminal edges, let $W^{\prime}=\left\{K \in V\left(\mathcal{Q}_{N-1}(G)\right): \mathrm{e}_{K}\right.$ is a terminal edge of $\left.G\right\}$; otherwise, let $W^{\prime}=\varnothing$.

If $W^{\prime} \neq \varnothing$, then by Theorem 65 , every vertex of $W^{\prime}$ is adjacent to all the vertices of $\mathcal{Q}_{N-1}(G)$. This implies that $\mathcal{Q}_{N-1}(G)$ is stitched.

If $W^{\prime}=\varnothing$, since we are assuming that $G$ is not a cycle, then $W \neq V\left(\mathcal{Q}_{N-1}(G)\right)$. So, $V=V\left(\mathcal{Q}_{N-1}(G)\right)-W$ is not empty.

Let $K \in W$ and let $L \in V$. We will show that $K$ and $L$ are level adjacent. 
Since $K \in W, \mathrm{e}_{K}$ is an edge of $C_{0}$. Since $L \notin W, \mathrm{e}_{L}$ is a cycle edge not in $C_{0}$. Using Theorem 67, we have that $K$ and $L$ are level adjacent.

Therefore, by Remark $71, \mathcal{Q}_{N-1}(G)$ is stitched.

On the other hand, let $G$ be a cycle of size greater than 4 . Then, by Theorem $53, \mathcal{Q}_{N-1}(G)$ is a cycle with size greater than 4 ; therefore, $\mathcal{Q}_{N-1}(G)$ does not contain a spanning bipartite complete subgraph. This is since for any partition $V$ and $W$ of a cycle with at least five vertices, either $V$ of $W$ has at least three elements; therefore, if the cycle has a spanning complete bipartite subgraph, it has to contain a vertex of degree 3 . This is not possible.

\subsection{DEGREES IN THE SIZE LEVELS.}

First, we prove a theorem that characterizes the subgraphs that are level adjacent to a given subgraph. This makes counting the edges incident to a given subgraph in the size level easy.

Theorem 73 Let $G$ be a connected graph and let $K$ be a connected nondegenerate subgraph of $G$ with size $n$. The subgraph $L$ of $G$ is level adjacent to $K$ if and only if one of the following holds:

(1) There is an endvertex $v$ of $K$ and $\mathrm{e} \in E(K-\{v\}, G)$ such that $L=(K-\{v\}) \cup \mathrm{e}$.

(2) There is a cycle edge $\mathrm{f}$ of $K$ and $\mathrm{e} \in E(K, G)$ such that $L=(K-\mathrm{f}) \cup \mathrm{e}$.

Proof. Let $L$ be a subgraph of $G$ level adjacent to $K$, let $C=\operatorname{core}(K, L)$, let $\mathrm{e} \in E(L)-E(K)$ and let $\mathrm{f} \in E(K)-E(L)$. 
Note that $\mathrm{f}$ is the complementary edge set of $C$ in $K$. By Lemma 32, there is an endvertex $v$ of $K$ such that $C=K-\{v\}$ if $\mathbf{f}$ is a terminal edge or such that $C=K-\mathrm{f}$ if $\mathrm{f}$ is a cycle edge of $K$. In either case, since $L$ is connected, e is incident with $C$. Then $C \cup \mathrm{e}$ is a connected subgraph of $G$ having $\mathrm{f}$ has its complementary edge in $j \operatorname{oin}(K, L)$. By Remark 31, $L=C \cup \mathrm{e}$.

On the other hand, let $C=K-\{v\}$ if we assume (1) or let $C=K-\mathrm{f}$ if we assume (2). In any case $C$ is a connected subgraph of both $K$ and $L$ with size $n-1$, therefore $K$ and $L$ are level adjacent.

As a consequence of this theorem, every subgraph $L$ level adjacent to $K$ can be associated with a unique pair $(e, f)$, where e is an edge incident with $K$ and $f$ is a removable edge of $K$. Note that this association is not surjective since, when $\mathrm{f}$ is a terminal edge with endvertex $v$ and e is incident with $v,(K-\{v\}) \cup \mathrm{e}$ is not connected.

Corollary 74 Let $G$ be a connected graph, let $K$ be a connected subgraph of $G$ with size $n \geq 1$, let $c$ be the number of cycle edges of $K$, let $d=|E(K, G)|$, let $T$ be the set of endvertices of $K$ and let $l(v)=|E(K-\{v\}, G)|$. Then

$$
\operatorname{deg}_{\mathcal{Q}_{n}(G)} K=c \cdot d+\sum_{v \in T} l(v)
$$

Proof. By Theorem 73, every subgraph level adjacent to $K$ is either like in part (1) or like in part (2) of that theorem. The number of possible subgraphs $L$ of the form $L=(K-\mathrm{f}) \cup \mathrm{e}$ where $\mathrm{f}$ is a cycle edge of $K$ and $\mathrm{e} \in E(K, G)$ is $c \cdot d$. The number of possible subgraphs $L$ of the form $L=(K-\{v\}) \cup$ e where $v$ is an endvertex of $K$ and $\mathrm{e} \in E(K-\{v\}, G)$ is $\sum_{v \in T} l(v)$. 
Corollary 75 Let $G$ be a connected graph and let $K$ be a connected subgraph of $G$ with size $n$. If $K$ contains a cycle or $K$ has at least 4 endpoints, then $\operatorname{deg}_{\mathcal{Q}_{n}(G)} K \geq 3$.

Proof. Just use the formula in Corollary 74.

\subsection{NEIGHBORHOODS AND CUT VERTICES}

Now, we analyze the neighborhoods in the size levels. We also characterize the subgraphs that are cutvertices of the levels in Theorem 91. First, we give the definition of neighborhood.

Definition 76 Let $G$ be a graph and $v$ be a vertex of $G$. The neighbors of $v$ in $G$, denoted by $N_{G}(v)$, is the set of all the vertices of $G$ that are adjacent to $v$.

$$
N_{G}(v)=\{w \in V(G): v \text { and } w \text { are adjacent }\}
$$

Observe that $\operatorname{deg}_{G} v=\left|N_{G}(v)\right|$.

In our particular case, given a size level and a vertex of such size level, we are interested in the subgraph of the size level induced by the neighbors of the vertex.

Definition 77 Let $G$ be a graph and let $v$ be a vertex of $G$. The neighborhood of $v$ in $G$ is the subgraph of $G$ induced by the vertex set $N_{G}(v)$. If $K$ is a connected subgraph of $G$ with size $n$, we denote the neighborhood of $K$ in $\mathcal{Q}_{n}(G)$ by $\mathcal{N}_{K}^{n}$.

Now, we analyze the connectedness of the neighborhoods of trees in the size levels. 
Lemma 78 Let $G$ be a connected graph, let $K$ be a proper subtree of $G$ with size $n \geq 2$, let $J$ and $L$ be two subgraphs that are level adjacent to $K$, let $C=\operatorname{core}(K, J)$, let $D=\operatorname{core}(K, L)$, let $\mathrm{e}_{C, J}$ be the complementary edge of $C$ in $J$ and let $\mathbf{e}_{D, L}$ be the complementary edge of $D$ in L. If $\mathrm{e}_{C, J}$ and $\mathrm{e}_{D, L}$ are incident with $K$ at the same vertex, then there is a path in $\mathcal{N}_{K}^{n}$ of length 2 or less joining $J$ and $L$.

Proof. Let $v$ be the vertex of $K$ such that $\mathbf{e}_{C, J}$ and $\mathbf{e}_{D, L}$ are incident to $v$. If $C=D$, then $J$ and $L$ are level adjacent.

So, let us assume that $C \neq D$. Both $C$ and $D$ are in $\mathcal{Q}_{n-1}(K)$ and $K$ is a tree. By Corollary 66, $C$ and $D$ are level adjacent. Also, note that both $C$ and $D$ have $v$ as a vertex, then $v$ is a vertex of core $(C, D)$ (recall Definition 41).

Define $L^{\prime}=C \cup \mathrm{e}_{D, L}$.

The graphs $J, K$ and $L^{\prime}$ contain $C$, then $J, K$ and $L^{\prime}$ are pairwise level adjacent. So, $J$ and $K^{\prime}$ are adjacent in $\mathcal{N}_{K}^{n}$.

Now, the graph core $(C, D) \cup \mathrm{e}_{D, L}$ is a connected subgraph of both $L$ and $L^{\prime}$ (since $\mathrm{e}_{D, L}$ is incident with $\operatorname{core}(C, D)$ at $\left.v\right)$. This implies that $L$ and $L^{\prime}$ are adjacent in $\mathcal{N}_{K}^{n}$.

Therefore, $J L^{\prime} L$ is a path of length 2 in $\mathcal{N}_{K}^{n}$.

Lemma 79 Let $G$ a connected graph and let $K$ be a proper subtree of $G$ with size $n \geq 2$. If there is an edge e incident with an interior vertex of $K$, then any two vertices of $\mathcal{N}_{K}^{n}$ can be joined by a path of length 4 or less in $\mathcal{N}_{K}^{n}$.

Proof. Let $J$ and $L$ be any two distinct subgraphs that are level adjacent to $K$, let $C=\operatorname{core}(K, J)$ and let $D=\operatorname{core}(K, L)$. By Remark 27 and Lemma 28, e is incident 
with $C$ and $D$. Define $J^{\prime}=\operatorname{core}(K, J) \cup$ e and $L^{\prime}=\operatorname{core}(K, L) \cup$ e. By construction, $J^{\prime}$ is level adjacent to both $K$ and $J$. We also have that $L^{\prime}$ is level adjacent to both $K$ and $L$. Thus, $J^{\prime}$ and $L^{\prime}$ are level adjacent to $K$. However, using Lemma 78, we see that there is a path of length 2 or less joining $J^{\prime}$ and $L^{\prime}$ in $\mathcal{N}_{K}^{n}$. Therefore, there is a path of length 4 or less joining $J$ and $K$ in $\mathcal{N}_{K}^{n}$.

Lemma 80 Let $K$ be a tree that is not a path. If $J$ and $L$ are level adjacent to $K$, then $J$ and $K$ are connected by a path in $\mathcal{N}_{K}^{n}$.

Proof. Let $C=\operatorname{core}(K, J), D=\operatorname{core}(K, L)$, let $\mathrm{e}_{C, J}$ and $\mathrm{e}_{D, L}$ be the complementary edges of $C$ in $J$ and $D$ in $L$, respectively. Let $v_{C, J}$ and $v_{D, L}$ be the vertices of $C$ and $D$ to which $\mathrm{e}_{C, J}$ and $\mathrm{e}_{D, L}$ are incident, respectively. Since $K$ is a tree that is not a path, $K$ has at least three endvertices. Then there is a connected subgraph $B$ of $K$ with size $n-1$ such that $v_{C, J}$ and $v_{D, L}$ are vertices of $B$. Define $J^{\prime}=B \cup \mathrm{e}_{C, J}$ and $K^{\prime}=B \cup \mathrm{e}_{D, L}$. Note that $J^{\prime}$ and $L^{\prime}$ are either level adjacent or equal (if $\mathrm{e}_{C, J}=\mathrm{e}_{D, L}$ ) and that both are level adjacent to $K$. Applying Lemma 78 to both pairs $J$ and $J^{\prime}$, and $L$ and $L^{\prime}$, we can conclude that both pairs can be joined by a path in $\mathcal{N}_{K}^{n}$ with length 2 or less. Hence, there is a walk in $\mathcal{N}_{K}^{n}$ joining $J$ and $L$; thus, there is a path joining $J$ and $L$ in $\mathcal{N}_{K}^{n}$.

Definition 81 Let $G$ be a graph and $K$ be a connected subgraph of $G$. The graph $K$ is a free subgraph in $G$ if every edge incident with $K$ is incident with endvertices of $K$ only.

Theorem 82 Let $K$ be a tree. If $K$ is not a free path, then $\mathcal{N}_{K}^{n}$ is connected. 
Proof. Suppose that $K$ is not a path. By Lemma $80, \mathcal{N}_{K}^{n}$ is connected. If $K$ is not a free subgraph, then by Lemma $79, \mathcal{N}_{K}^{n}$ is also connected.

Next, we analyze the neighborhoods of graphs that contain a cycle. To prove the next lemma, we need a definition.

Definition 83 Let $G$ be a connected graph and let $K$ and $L$ be two subgraphs of $G$. The graph $G$ is irreducible between $H$ and $K$ if no proper connected subgraph of $G$ contains both $K$ and $L$ as subgraphs.

Remark 84 Let $G$ be a connected graph. The graph $G$ is irreducible between $K$ and $L$ if and only if every cycle edge of $G$ is an edge of $K \cup L$ and every endvertex of $G$ is a vertex of $K \cup L$.

Lemma 85 Let $K$ be a graph containing a cycle $Y$ and let $J$ and $L$ be both level adjacent to $K$. Assume the following: $C=$ core $(K, J) ; D=\operatorname{core}(K, L) ; \mathrm{e}_{C, K}$ is the complementary edge of $C$ in $K$; $\mathrm{e}_{D, K}$ the complementary edge of $D$ in $K$ such that $\mathbf{e}_{C, K}$ and $\mathbf{e}_{D, K}$ are edges of the cycle $Y$. Then $J$ and $L$ are connected by a path in $\mathcal{N}_{K}^{n}$.

Proof. If $\mathrm{e}_{C, K}=\mathrm{e}_{D, K}$, then $K-\mathrm{e}_{C, K}$ is a connected graph contained in both $J$ and $L$. Hence, $J$ and $L$ are level adjacent.

Therefore, we can assume $\mathrm{e}_{C, K} \neq \mathrm{e}_{D, K}$.

Let $\mathrm{f}_{C, J}$ be the complementary edge of $C$ in $J$, let $v_{C, J}$ be a vertex of both e $_{C, J}$ and $C$ and let $\mathrm{f}_{D, L}$ be the complementary edge of $D$ in $L$. We use the letter $\mathrm{f}$ to describe the edges that are incident to $K$ and the letter e for the edges that are in $K$. 
Note that $\mathrm{e}_{C, K}$ and $\mathrm{e}_{D, K}$ are cycle edges of $K$. By Remark 27 and Lemma 32, we have that $C=K-\mathrm{e}_{C, K}$ and $D=K-\mathrm{e}_{D, K}$. Moreover, $\mathbf{f}_{C, J}$ is incident with $D$ and $\mathbf{f}_{D, L}$ is incident with $C$. Thus, $\mathbf{f}_{C, J}$ and $\mathbf{f}_{D, L}$ are incident with $K$. Let $\left\{v_{C, J}\right\}$ and $\left\{v_{D, L}\right\}$ be the vertices of $K$ that are vertices of $\mathbf{f}_{C, J}$ and $\mathbf{f}_{D, L}$, respectively.

We consider two cases:

CASE 1. $K$ is not irreducible between $Y$ and $\left\{v_{D, L}\right\}$.

Without loss of generality, assume there is a connected subgraph $E$ of $K$ with size $n-1$ such that the cycle $Y$ and $\left\{v_{D, L}\right\}$ are contained in $E$. Then $\mathbf{f}_{D, L}$ is adjacent to $E$. Let e be the complementary edge of $E$ in $K$.

Define $J^{\prime}=C \cup \mathbf{f}_{D, L}$ (Figure $3.2(\mathrm{~d})$ ). Then $J^{\prime}$ is a connected subgraph with size $n$. Since $C$ is a subgraph of $K, J$ and $J^{\prime} ; J^{\prime}$ is level adjacent to both $J$ and $K$.

Define now $L^{\prime}=E \cup \mathbf{f}_{D, L}$ (Figure $3.2(\mathrm{e})$ ). The graph $E$ is contained in both $K$ and $L^{\prime}$, so $K$ and $L^{\prime}$ are level adjacent. Since $j \operatorname{oin}(K, L)=K \cup \mathrm{f}_{D, L}, L^{\prime}$ is a subgraph of join $(K, L)$. Note that e is the complementary edge of $L^{\prime}$ in join $(K, L)$, Hence, applying Theorem 67 to the subgraph $L$ and the cycle $Y$ in $j \operatorname{oin}(K, L)$, we see that $L$ and $L^{\prime}$ are level adjacent in $\mathcal{Q}_{n}(j \operatorname{oin}(K, L))$ and, therefore, in $\mathcal{Q}_{n}(G)$. Thus, $J J^{\prime} L^{\prime} L$ is a path in $\mathcal{N}_{K}^{n}$. The same argument works when $K$ is not irreducible between $Y$ and $\left\{v_{D, L}\right\}$.

CASE 2. $K$ is irreducible between $K$ and $\left\{v_{D, L}\right\}$.

By Remark 84, the only removable edges of $K$ are edges of $Y$, or perhaps, one terminal edge of $K$ incident with $v_{D, L}$. In other words, $K$ is such that every edge outside of $Y$ is a bridge and $K$ has at most one endpoint, namely $v_{D, L}$. Then $K=Y$ or $K$ is the result of attaching a path to $Y$ at one vertex. 
Since $Y$ is a cycle, we can find a path $P=\mathrm{e}_{1} \mathrm{e}_{2} \ldots \mathrm{e}_{l}$ of $Y$ such that: $P$ is a free path in $K ; \mathrm{e}_{1}=\mathrm{e}_{C, K} ; \mathrm{e}_{l}=\mathrm{e}_{D, K} ; v_{D, L}$ is not an interior vertex of $P$.

Define $J_{i}=\left(K-\mathrm{e}_{i}\right) \cup \mathrm{f}_{D, L}$. Since $\mathrm{e}_{i}$ is a cycle edge of $K, \mathrm{f}_{D, L}$ is incident with $K-\mathrm{e}_{i}$; hence, $J_{i}$ is connected for all $i$ and is level adjacent to $K$.

Let $v_{i}$ be the common vertex between $\mathrm{e}_{i}$ and $\mathrm{e}_{i+1}$. Since $P$ is free in $K, K-\left\{v_{i}\right\}$ is a connected subgraph of $K$ with size $n-2$. Observe that $\left\{\mathbf{e}_{i}, \mathbf{e}_{i+1}\right\}$ is the complementary edge set of $K-\left\{v_{i}\right\}$ in $K$. But, $K-\left\{v_{i}\right\}$ is contained in both $J_{i}$ and $J_{i+1}$; it follows that $J_{i}$ and $J_{i+1}$ are level adjacent for all $i$.

Note that $\mathrm{e}_{C, K}$ is the complementary edge of both $K-\mathrm{e}_{1}$ and $C$ in $K$, hence, $K-\mathrm{f}_{1}=C$. Thus, $J_{1}=J$.

Observe that $K-\mathrm{e}_{l}$ is a connected graph contained in both $J_{l}$ and $L$; thus, $J_{l}$ and $L$ are level adjacent.

Therefore, $J J_{1} J_{2} \ldots J_{l} L$ is a path in $\mathcal{N}_{K}^{n}$.

Lemma 86 If $K$ and $L$ are level adjacent subgraphs such that both contain the cycle $Y$, then for every edge e of $Y$ there is a connected graph $L_{\mathbf{e}}$ such that e is not an edge of $L_{\mathbf{e}}$ and $L_{\mathrm{e}}$ is level adjacent to both $K$ and $L$.

Proof. Let $C=\operatorname{core}(K, L)$, let e be an edge of $Y$ and let $\mathrm{e}_{C, L}$ be the complementary edge of $C$ in $L$. Note that $\mathrm{e}_{C, L}$ is incident with $K-\mathrm{e}$. Define $L_{\mathrm{e}}=(K-\mathrm{e}) \cup \mathrm{e}_{C, L}$. 


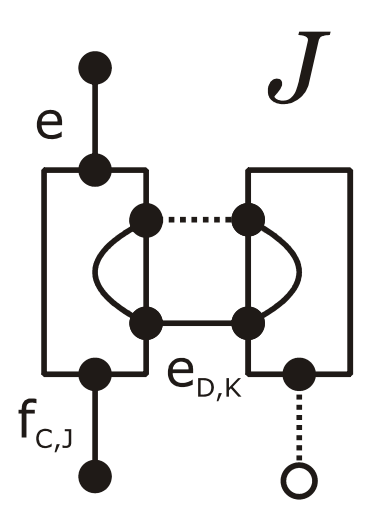

(a)

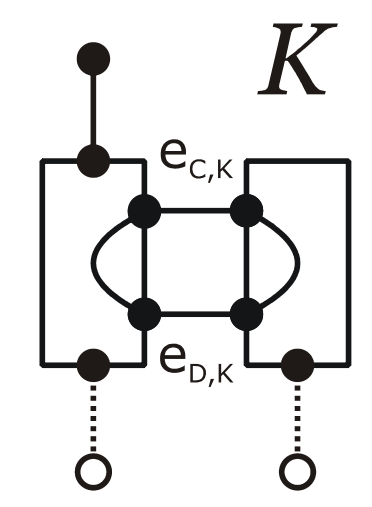

(b)

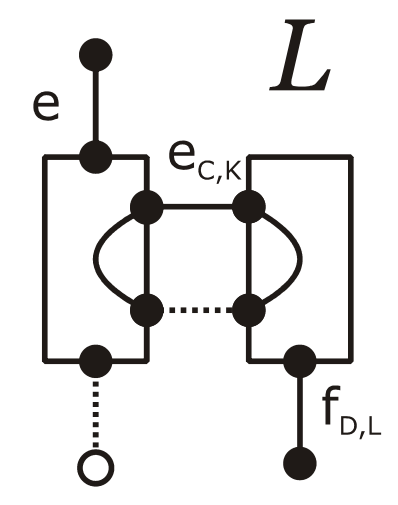

(c)

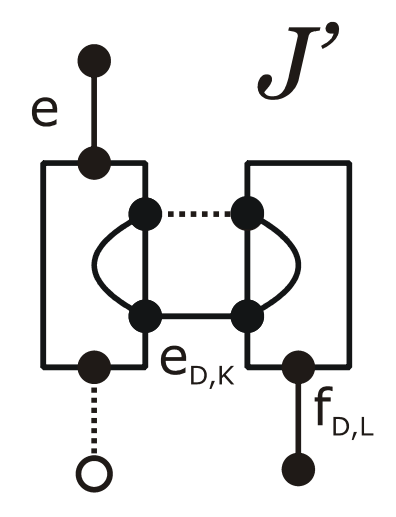

(d)

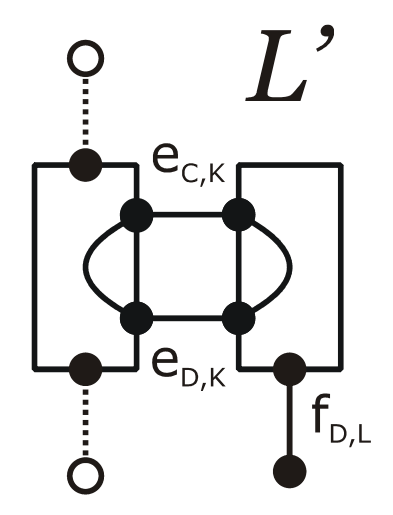

(e)

Figure 3.2: Graphs in the first case of Lemma 85 
By using Theorem 67 applied to $L_{\mathrm{e}}$ in $\operatorname{join}(K, L)$, we have that $L_{\mathrm{e}}$ is level adjacent to both $K$ and $L$.

Theorem 87 Let $K$ be a connected proper subgraph of $G$ with size $n$. If $K$ contains a cycle, then $\mathcal{N}_{K}^{n}$ is connected.

Proof. Let $J$ and $K$ be two vertices of $\mathcal{N}_{K}^{n}$, let $C=\operatorname{core}(K, J)$ and let $D=$ $\operatorname{core}(K, L)$. We show that $J$ and $K$ are connected by a path in $\mathcal{N}_{K}^{n}$.

Let $\mathrm{e}_{C, K}$ be the complementary edge of $C$ in $K$, let $\mathrm{e}_{D, K}$ be the complementary edge of $D$ in $K$ and let $Y$ be a cycle contained in $K$.

If $\mathrm{e}_{C, K}$ and $\mathrm{e}_{D, K}$ are both contained in $Y$, then by Lemma $85, J$ and $L$ are connected by a path in $\mathcal{N}_{K}^{n}$.

So, assume that $\mathrm{e}_{C, K}$ is not contained in $Y$. Hence, $Y$ is a subgraph of $J$. Take e an edge of $Y$. Using Lemma 86, there is a subgraph $J_{\mathrm{e}}$ of $G$ such that $J_{\mathrm{e}}$ does not have e as an edge and $J_{\mathrm{e}}$ is level adjacent to both $J$ and $K$. Hence, $J_{\mathrm{e}}$ is a vertex of $\mathcal{N}_{K}^{n}$ that is level adjacent to $J$.

If $\mathrm{e}_{D, K}$ is contained in $Y$, then applying Lemma $85, J_{\mathrm{e}}$ and $L$ are connected by a path in $\mathcal{N}_{K}^{n}$. This implies that $J$ and $L$ are connected by a path in $\mathcal{N}_{K}^{n}$.

If $\mathrm{e}_{D, K}$ is not contained in $Y$, then by an argument similar to the one for $J$, there is a subgraph $L_{\mathrm{e}}$ of $G$ such that $L_{\mathrm{e}}$ is a vertex of $\mathcal{N}_{K}^{n}$ that is level adjacent to $L$. But note that $J_{\mathrm{e}}$ and $L_{\mathrm{e}}$ are level adjacent. Therefore, $J J_{\mathrm{e}} L_{\mathrm{e}} L$ is a path in $\mathcal{N}_{K}^{n}$.

Theorem 88 Let $G$ be a connected graph and let $K$ be a connected subgraph of $G$. If $K$ is not a free path, then $\mathcal{N}_{K}^{n}$ is connected. 
Proof. Assume that $\mathcal{N}_{K}^{n}$ is not connected. Hence, by Theorem $87, K$ is a tree. Thus, by Theorem $82, K$ has to be a free path.

Corollary 89 Let $G$ be a connected graph and let $K$ be a connected subgraph of $G$. If $K$ is not a free path, then $K$ is not a cutvertex of $\mathcal{Q}_{n}(G)$.

Hence, if we have a cut-vertex in a size level, then it has to be a free path. But not all the free paths are cut-vertices of the size levels, as it is shown in the following lemma:

Lemma 90 Let $G$ be a connected graph and let $K$ be a free path. If $K$ is contained in a cycle, then $K$ is not a cut-vertex of $\mathcal{Q}_{n}(G)$.

Proof. Assume that $K$ is a cut-vertex of $Q_{n}(G)$. Then there is a separation $\mathcal{M}$ and $\mathcal{N}$ of $\mathcal{Q}_{n}(G)-\{K\}$. Since $K$ is a path, there are exactly two subgraphs of $K$, namely $C$ and $D$. Note that since $K$ is a cut-vertex, $\mathcal{N}_{K}^{n}$ is not connected.

Define

$\mathcal{C}=\left\langle L \in V\left(\mathcal{N}_{K}^{n}\right): C=\operatorname{core}(K, L)\right\rangle$ and

$\mathcal{D}=\left\langle L \in V\left(\mathcal{N}_{K}^{n}\right): D=\operatorname{core}(K, L)\right\rangle$

Note that $\mathcal{C}$ and $\mathcal{D}$ are connected subgraphs of $\mathcal{Q}_{n}(G)-\{K\}$. But $\mathcal{M}$ and $\mathcal{N}$ is a separation of $\mathcal{Q}_{n}(G)-\{K\}$ and $\mathcal{Q}_{n}(G)$ is connected, so $\mathcal{C}$ and $\mathcal{D}$ are contained in distinct elements of the separation. Without loss of generality, assume that $\mathcal{C} \subset \mathcal{M}$ and $\mathcal{D} \subset \mathcal{N}$.

Let $Y$ be the cycle that contains $K$. As a consequence of Theorem $53, \mathcal{Q}_{n}(Y)$ is a cycle. So, there are $J$ and $L$ two subgraphs of $Y$ such that $J$ and $L$ are paths and $K$ is level adjacent to $J$ and $L$. Assume that $J$ is a vertex of $\mathcal{C}$ and $L$ is a vertex of $\mathcal{D}$. Observe that 
$J$ and $L$ are joined by a path not containing $K$ in $\mathcal{Q}_{n}(Y)$ and therefore in $\mathcal{Q}_{n}(G)-\{K\}$. This is a contradiction to the fact that that $\mathcal{M}$ and $\mathcal{N}$ is a separation.

We are ready to characterize the cutvertices of the size levels. Note that if $K$ is a free path, with endvertices $v$ and $w$ such that $K$ is not contained in a cycle, then $G-(V(K)-\{v, w\})$ is a disconnected graph. Moreover, $G-(V(K)-\{v, w\})$ has exactly two components.

Theorem 91 Let $G$ be a connected graph and let $K$ be a connected subgraph of $G$ with size $n \geq 1$. Then $K$ is a cut-vertex of $\mathcal{Q}_{n}(G)$ if and only if $K$ is a free path such that $K$ does not contain an endvertex of $G$ and $K$ is not contained in a cycle.

Proof. If $K$ is a cutvertex of $\mathcal{Q}_{n}(G)$, then by Corollary $89, K$ has to be a free path. Using Lemma 90, we see that $K$ is not contained in a cycle.

On the other hand, assume that $K$ is a free path that is not contained in a cycle with endvertices $v$ and $w$. Let $C=K-\{v\}$ and $D=K-\{w\}$ be the only two subgraphs of $K$.

Let $F=G-(V(K)-\{v, w\})$, let $M$ and $N$ be a separation of $F$. Since $v$ and $w$ are not endvertices, $M \neq\{v\}$ and $N \neq\{w\}$.

CLAIM 1. For any connected subgraph $L$ of $G$ such that $V(L) \cap V(M) \neq \varnothing$ and $V(L) \cap V(N) \neq \varnothing$ implies that $K$ is a subgraph of $L$.

Let e be an edge of $K$. Note that $G$ - e is disconnected with separation $M^{\prime}$ and $N^{\prime}$. Therefore, $M \subset M^{\prime}$ and $N \subset N^{\prime}$. Note that $L$ is connected, $V(L) \cap V\left(M^{\prime}\right) \neq \varnothing$ and $V(L) \cap V\left(N^{\prime}\right) \neq \varnothing$; thus, $L$ is not a subgraph of $G-$ e. Hence, e is an edge of $L$. This 
implies that $K$ is a subgraph of $L$.

So, $K$ is the only subgraph of $G$ of size $n$ or less such that $V(L) \cap V(M) \neq \varnothing$ and $V(L) \cap V(N) \neq \varnothing$.

Let $v^{\prime} \in V(M)$ and $w^{\prime} \in V(N)$. Hence, there are connected subgraphs $H^{\prime}$ and $J^{\prime}$ of $G$ with size $n$ such that $v^{\prime} \in V\left(H^{\prime}\right)$ and $w^{\prime} \in V\left(J^{\prime}\right)$. Therefore

$$
\begin{array}{r}
\left\{J \in V\left(\mathcal{Q}_{n}(G)-\{K\}\right): V(J) \cap V(M) \neq \varnothing\right\} \neq \varnothing \text { and } \\
\left\{J \in V\left(\mathcal{Q}_{n}(G)-\{K\}\right): V(J) \cap V(N) \neq \varnothing\right\} \neq \varnothing .
\end{array}
$$

Define

$$
\begin{gathered}
\mathcal{M}=\left\langle J \in V\left(\mathcal{Q}_{n}(G)-\{K\}\right): V(J) \cap V(M) \neq \varnothing\right\rangle \text { and } \\
\mathcal{N}=\left\langle J \in V\left(\mathcal{Q}_{n}(G)-\{K\}\right): V(J) \cap V(N) \neq \varnothing\right\rangle .
\end{gathered}
$$

Every connected subgraph $J$ of $G$ with size $n$ distinct from $K$ is a vertex of $\mathcal{M}$ or a vertex of $\mathcal{N}$, but not of both. This means that $\mathcal{M}$ and $\mathcal{N}$ are vertex disjoint. If $J$ is level adjacent to $J^{\prime}$ in $\mathcal{Q}_{n}(G)$ and $J$ is a vertex of $\mathcal{M}$, then $\operatorname{core}\left(J, J^{\prime}\right)$ has a vertex in $M$. By CLAIM 1 , no vertex of core $\left(J, J^{\prime}\right)$ is in $N$. Thus, $J^{\prime}$ is a vertex in $\mathcal{M}$. The same argument can be used for the case when $J$ is in $\mathcal{N}$. Hence, $\mathcal{M} \cup \mathcal{N}=\mathcal{Q}_{n}(G)-\{K\}$. Thus, $\mathcal{M}$ and $\mathcal{N}$ is a separation of $\mathcal{Q}_{n}(G)-\{K\}$. Therefore, $K$ is a cutvertex.

\subsection{HAMILTONIAN LEVELS}

One important question that we mention in Chapter 1 was to determine when the hyperspace graph is hamiltonian. We discuss some particular cases in Section 4.2. This question leads us to ask when the size levels are hamiltonian. We give a partial answer to this question here. 
Definition 92 A graph $G$ is hamiltonian if it has a cycle containing every vertex of $G$. Such cycle is called a hamiltonian cycle of $G$.

Actually, we can ask a more general question: Given any graph, which nondegenerate size levels of a graph are hamiltonian?

We have three conjectures for the answer to the question we raised above:

Conjecture 93 If $G$ is a connected graph with size $N$ and $G$ is not a path, then $\mathcal{Q}_{N-1}(G)$ is hamiltonian.

Conjecture 94 Let $G$ be a connected graph. If every free path of size $n$ is contained in a cycle, then $\mathcal{Q}_{n}(G)$ is hamiltonian.

Conjecture 95 Let $G$ be a connected graph with size $N$. If $n \leq N-2$ and $\mathcal{Q}_{n}(G)$ is hamiltonian, then $\mathcal{Q}_{n+1}(G)$ is hamiltonian.

Of course, proving the third conjecture would show that being hamiltonian is a size property.

We can answer this question partially for the case when the original graph is a star. The proof is based in combinatorics. First, we define a special graph.

Definition 96 Define the graph $Q(k, n)$ as follows:

- The vertex set of $Q(k, n)$ consists of all the subsets of $\{1,2, \ldots, n\}$ with $k$ elements.

- Two vertices $A$ and $B$ of $Q(k, n)$ are adjacent if $A \cap B$ has $k-1$ elements. 
Since in a star every subset of the edge set induces a unique connected subgraph, we have that:

Remark 97 Let $S_{n}$ be a star with size $n$. Then $Q(k, n)$ is isomorphic to $\mathcal{Q}_{k}\left(S_{n}\right)$.

Observe that for every $n$ positive integer, $Q(1, n)$ and $Q(n-1, n)$ are isomorphic to the complete graph with $n$ vertices $\mathcal{K}_{n}$. The following proposition shows a symmetry of these graphs:

Proposition 98 The graphs $Q(k, n)$ and $Q(n-k, n)$ are isomorphic for every $n$ and every $k \leq \frac{n}{2}$

Proof. Let $Z=\{1,2, \ldots, n\}$ and let $\alpha: Q(k, n) \rightarrow Q(n-k, n)$ be function defined by $\alpha(A)=Z-A$ for each $A \in Q(k, n)$.

Clearly, $\alpha$ is a one-to-one onto function.

Let $A$ and $B$ be two adjacent vertices in $Q(k, n)$. Note that $|\alpha(A)|=|\alpha(B)|=n-k$ and $\alpha(A) \cap \alpha(B)=Z-(A \cup B)$. Using that $|A \cap B|=k-1$ we have:

$$
|\alpha(A) \cap \alpha(B)|=|Z-(A \cup B)|=n-(k+1)=(n-k)-1 .
$$

Therefore, $\alpha$ is an isomorphism.

Given $Q(k, n)$ and $Q(k+1, n)$, define $[Q(k, n), Q(k+1, n)]$ by:

$$
\begin{gathered}
{[Q(k, n), Q(k+1, n)]=} \\
Q(k, n) \cup Q(k+1, n) \cup\langle A B:|A|=k,|B|=k+1 \text { and } A \subset B\rangle .
\end{gathered}
$$

Define $\phi: V([Q(k, n), Q(k+1, n)]) \rightarrow V(Q(n+1, k+1))$ by: 


$$
\phi(A)=\left\{\begin{array}{cc}
A \cup\{n+1\} & \text { if } A \in V(Q(k, n)) \\
A & \text { if } A \in V(Q(k+1, n))
\end{array} .\right.
$$

Theorem $99 \phi$ is an isomorphism between graphs and therefore $Q(k+1, n+1)$ is isomorphic to $[Q(k, n), Q(k+1, n)]$.

Proof. Note that the function $\phi$ is well-defined.

We prove that $\phi$ is one-to-one.

Let $A, B \in V([Q(k, n), Q(k+1, n)])$ be such that $\phi(A)=\phi(B)$.

If $n+1 \notin \phi(A)$, then $A, B \in V(Q(k+1, n))$. Thus, $A=B$.

If $n+1 \in \phi(A)$, then $n+1 \notin A$ and $n+1 \notin B$. Thus, $A=\phi(A)-\{n+1\}$ and $B=\phi(B)-\{n+1\}$. Since $\phi(A)=\phi(B)$, we have that $A=B$.

We prove that $\phi$ maps onto $[Q(k, n), Q(k+1, n)]$.

Let $C \in V(Q(n+1, k+1))$. If $n+1 \notin C$, then $C \in V(Q(k+1, n))$. Thus, $\phi(C)=C$.

If $n+1 \in C$, then define $A=C-\{n+1\}$. Observe that $A \in V(Q(k, n))$ and that $\phi(A)=C$.

We prove that $\phi$ preserves adjacency. Let $A$ and $B$ be adjacent in $[Q(k, n), Q(k+1, n)]$. If $A$ and $B$ are adjacent in $Q(k, n)$, then $|\phi(A) \cap \phi(B)|=|A \cap B|+1=k$.

If $A$ and $B$ are adjacent in $Q(k+1, n)$, then $|\phi(A) \cap \phi(B)|=|A \cap B|=k$.

Without loss of generality, assume that $A \in V(Q(k, n))$ and $B \in V(Q(k+1, n))$.

Then $A \subset B$. Thus, $\phi(A) \cap \phi(B)=(A \cup\{n+1\}) \cap B=A$. Therefore, $|\phi(A) \cap \phi(B)|=k$. 
We need to define a property that is stronger than being hamiltonian

Definition 100 A graph $G$ is strongly hamiltonian if for every edge $G$ there exists a hamiltonian cycle containing that edge.

Note that every strongly hamiltonian graph is hamiltonian; however, the converse is false: the graph consisting of a cycle and a chord is hamiltonian but not strongly hamiltonian.

Theorem $101 Q(k, n)$ is a strongly hamiltonian graph for every $n \geq 3$ and every $k<n$.

Proof. The proof will be done by induction over $n$.

If $n=3$, then $Q(1,3)$ and $Q(2,3)$ are isomorphic to $\mathcal{K}_{3}$. Therefore, $Q(1,3)$ and $Q(2,3)$ are strongly hamiltonian.

Assume that for $n=m, Q(k, m)$ is strongly hamiltonian for every $k$.

We show that $Q(k, m+1)$ is strongly hamiltonian.

Note that $Q(1, m+1)$ and $Q(m, m+1)$ are isomorphic to $\mathcal{K}_{m+1}$ and, therefore, are strongly hamiltonian.

Then, we can consider $2 \leq k \leq m-1$. By Theorem $99, Q(k, m+1)$ is isomorphic to $[Q(k-1, m), Q(k, m)]$. Therefore, it is sufficient to prove that $[Q(k-1, m), Q(k, m)]$ is strongly hamiltonian for all $k$ with $2 \leq k \leq m-1$.

Let $A$ and $A^{\prime}$ be two vertices of $[Q(k-1, m), Q(k, m)]$ such that $A$ and $A^{\prime}$ are adjacent in $[Q(k-1, m), Q(k, m)]$.

CASE 1. $A$ and $A^{\prime}$ are in $Q(k-1, m)$. 
By our induction assumption, $Q(k-1, m)$ is strongly hamiltonian. Hence, there is a hamiltonian cycle $\mathcal{L}_{1}$ in $Q(k-1, m)$ containing the edge $A A^{\prime}$. Let $B B^{\prime}$ be an edge of $\mathcal{L}_{1}$ distinct from $A A^{\prime}$. Define $\mathcal{P}_{1}$ to be the path obtained by removing $B B^{\prime}$ from $\mathcal{L}_{1}$.

Let $c^{\prime} \in B^{\prime}-B$. Note that, since $k-1 \leq m-2$, there is $c \notin B$ such that $c \neq c^{\prime}$.

Observe also that $B \subset C, B \subset C^{\prime}$ and $B^{\prime} \subset C^{\prime}$. Thus, $C=B \cup\{c\}$ and $C^{\prime}=B \cup\left\{c^{\prime}\right\}$ are vertices satisfying:

1. $C$ and $C^{\prime}$ are adjacent in $Q(k, m)$;

2. $B$ and $C$ are adjacent in $[Q(k-1, m), Q(k, m)]$;

3. $B^{\prime}$ and $C^{\prime}$ are adjacent in $[Q(k-1, m), Q(k, m)]$.

Since $Q(k, m)$ is strongly hamiltonian, there is a hamiltonian cycle $\mathcal{L}_{2}$ in $Q(k, m)$ such that $\mathcal{L}_{2}$ contains the edge $C C^{\prime}$. Let $\mathcal{P}_{2}$ be the path obtained by removing $C C^{\prime}$ from $\mathcal{L}_{2}$.

Then $\mathcal{L}=\mathcal{P}_{1} \cup B C \cup \mathcal{P}_{2} \cup B^{\prime} C^{\prime}$ is a hamiltonian cycle in $[Q(k-1, m), Q(k, m)]$ containing the edge $A A^{\prime}$. For the case when $A$ and $A^{\prime}$ are in $Q(k, m)$, a hamiltonian cycle in $[Q(k-1, m), Q(k, m)]$ can be constructed in a similar way.

CASE 2. $A$ is a vertex in $Q(k-1, m)$ and $A^{\prime}$ is a vertex in $Q(k, m)$.

Note that in this case $A \subset A^{\prime}$. Since $k \leq m-1$, there is $b \in\{1,2, \ldots, m\}-A^{\prime}$. Let $a \in A$. Define $B^{\prime}=A \cup\{b\}$ and $B=B^{\prime}-\{a\}$. Since $A \cap B=A-\{a\}, A \subset A^{\prime}, A \subset B^{\prime}$, we see that:

1. $A$ and $B$ are adjacent in $Q(k-1, m)$; 
2. $A^{\prime}$ and $B^{\prime}$ are adjacent in $Q(k, m)$;

3. $B$ and $B^{\prime}$ are adjacent in $[Q(k-1, m), Q(k, m)]$.

Again, by our induction assumption, $Q(k-1, m)$ and $Q(k, m)$ are strongly hamiltonian. There is a hamiltonian cycle $\mathcal{L}_{1}$ in $Q(k-1, m)$ containing the edge $A B$ and there is a hamiltonian cycle $\mathcal{L}_{2}$ in $Q(k, m)$ such that $\mathcal{L}_{2}$ contains the edge $A^{\prime} B^{\prime}$. Let $\mathcal{P}_{1}$ be the path obtained by removing $A B$ from $\mathcal{L}_{1}$ and $\mathcal{P}_{2}$ be the path obtained by removing $A^{\prime} B^{\prime}$ from $\mathcal{L}_{2}$. Note that the endvertices of such paths are $A$ and $B$ for $\mathcal{P}_{1}$ and $A^{\prime}$ and $B^{\prime}$ for $\mathcal{P}_{2}$.

Then $\mathcal{L}=\mathcal{P}_{1} \cup A A^{\prime} \cup \mathcal{P}_{2} \cup B B^{\prime}$ is a hamiltonian cycle containing the edge $A A^{\prime}$.

Therefore, $[Q(k-1, m), Q(k, m)]$ is strongly hamiltonian and so is $Q(k, m+1)$.

We note two corollaries.

Corollary 102 All the nonzero, nondegenerate size levels of a star are strongly hamiltonian.

Corollary 103 Being hamiltonian is not size reversible. 


\section{Chapter 4}

\section{THE GRAPH $\mathcal{C}(\mathbf{G})$}

We begin Section 4.1 by defining the hyperspace graph $\mathcal{C}(G)$. We give a formula for the degrees of vertices in $\mathcal{C}(G)$ in Proposition 112. It is natural to ask for which graphs $G$, the hyperspace graph $\mathcal{C}(G)$ is planar. In Section 4.2 , we answer the question. Only paths, cycles and the 3-star have hyperspace graph $\mathcal{C}(G)$ planar (Theorem 118). We follow this result with related characterizations of paths, cycles and the 3-star in terms of hyperspace graphs (Theorems 121, 122 and 123). In Section 4.3, we use the results in the previous sections to show the following important and fundamental result: If two graphs have isomorphic hyperspace graphs, then the graphs are isomorphic. In the hyperspace of continua this result is known for topological graphs different from the arc and the simple close curve.

\subsection{DEFINITION AND BASIC PROPERTIES}

Definition 104 Let $G$ be a connected graph with size $N$. The hyperspace graph of connected subgraphs $\mathcal{C}(G)$ is the graph defined as follows: 
- The vertex set of $\mathcal{C}(G)$ is the set of all connected subgraphs of $G$. In other words:

$V(\mathcal{C}(G))=\{K$ connected subgraph of $G\}$

- Two connected subgraphs $K$ and $L$ are adjacent in $\mathcal{C}(G)$ if one of the following holds:

1. $K$ and $L$ are level adjacent ( $K$ and $L$ have the same size and are adjacent in the size level).

2. $K$ is a subgraph of $L$ and $|E(L)|=|E(K)|+1$.

The graph $\mathcal{C}(G)$ can be written as:

$$
\mathcal{C}(G)=\left(\bigcup_{n=0}^{N} \mathcal{Q}_{n}(G)\right) \cup\langle K L: K \subset L \text { and }|E(L)|=|E(K)|+1\rangle
$$

In Figure 4.1 we show some examples of $\mathcal{C}(G)$ when (a) $G$ is a path, (b1) the standard model when $G$ is a cycle of size 3, (b2) is the planar model; (c1) the standard model when $G$ is the 3 -star and (c2) is the planar model.

Here are some important properties of the graph $\mathcal{C}(G)$ :

Remark 105 Let $G$ be a connected graph.If $K$ and $L$ are two connected subgraphs of $G$ such that $K$ and $L$ are adjacent in $\mathcal{C}(G)$, then ||$E(K)|-| E(L)|| \leq 1$.

Remark 106 Every size level $\mathcal{Q}_{n}(G)$ is a connected subgraph of $\mathcal{C}(G)$.

Remark 107 If $K$ and $L$ are level adjacent subgraphs of $G$, then 



(b2)
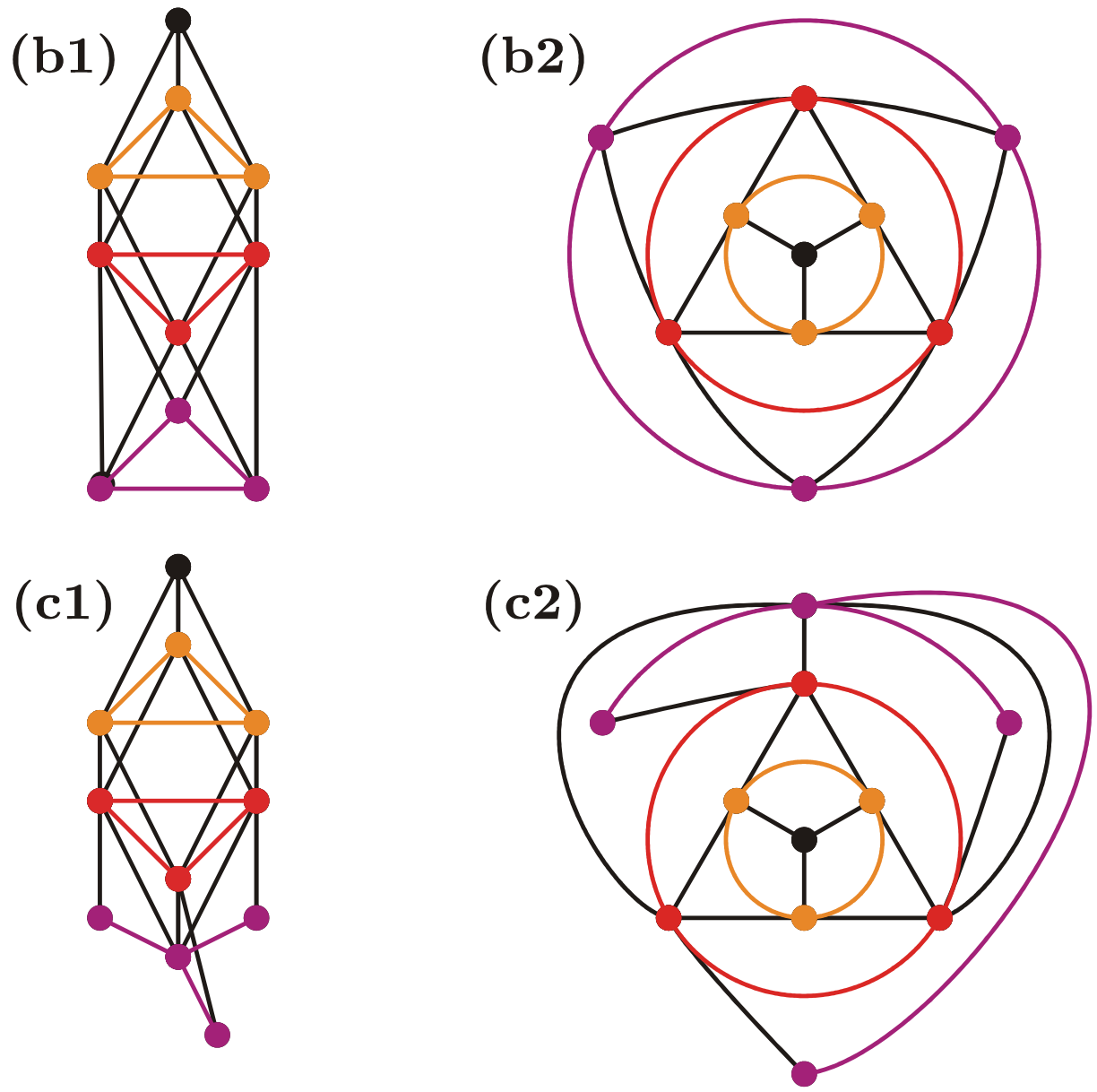

Figure 4.1: Examples of the graph $\mathcal{C}(G)$. 
1. $K$ and $L$ are adjacent in $\mathcal{C}(G)$;

2. $\operatorname{core}(K, L)$ is adjacent to both $K$ and $L$ in $\mathcal{C}(G)$;

3. $\operatorname{join}(K, L)$ is adjacent to both $K$ and $L$ in $\mathcal{C}(G)$.

As a consequence of the Lemma 35, we can make the following comment:

Remark 108 If $G$ is a connected graph of size $N$ and $K$ is a subgraph of $G$ with size $M$, then there is a path $\mathcal{P}=K_{0} K_{1} \ldots K_{N-M}$ in $\mathcal{C}(G)$ such that $K_{0}=K, K_{N-M}=G$. Moreover, the graph $K_{n}$ has size $M+n$ for every $n=0,1, \ldots, N-M$.

Definition 109 The path in Remark 108 is called ordered path.

Note that Remark 108 guarantees the existence of these paths starting at any connected subgraph of $G$. Then we can easily see that:

Remark 110 The graph $\mathcal{C}(G)$ is connected for all connected graphs.

Also, from Remark 38, we conclude that:

Remark 111 If $G$ is connected graph and $H$ is a connected subgraph of $G$, then $\mathcal{C}(H) \subset$ $\mathcal{C}(G)$

We can use the formula of the degree in the size levels in Corollary 74 to calculate the degree in $\mathcal{C}(G)$. 
Proposition 112 Let $G$ be a connected graph, let $K$ be a connected subgraph of $G$ with size $n \geq 1$, let $c$ be the number of cycle edges of $K$, let $d=|E(K, G)|$, let $T$ be the set of endvertices of $K$ and let $l(v)=|E(K-\{v\}, G)|$. Then

$$
\operatorname{deg}_{\mathcal{C}(G)} K=\left(c \cdot d+\sum_{v \in T} l(v)\right)+(c+|T|)+d
$$

Proof. The number of subgraphs of $G$ that are level adjacent to $K$ is $\operatorname{deg}_{\mathcal{Q}_{n}(G)} K=$ $c \cdot d+\sum_{v \in T} l(v)$. The number of subgraphs of $G$ with size $n-1$ contained in $K$, by Lemma 32 is the number of removable edges of $K$, in other words, the total number of cycle edges and terminal edges. That number is $c+|T|$. Finally, the number of subgraphs of $G$ containing $K$ is just the number of edges incident with $K$, namely $d$.

\subsection{PLANARITY OF $\mathcal{C}(\mathrm{G})$}

In this section we characterize the graphs $G$ whose corresponding $\mathcal{C}(G)$ is planar.

First, some definitions:

Definition 113 The graph $G^{\prime}$ is an elementary subdivision of $G$ if $G^{\prime}$ can be obtained by removing an edge $\mathrm{e}=v v^{\prime}$ from $G$ and adding a new vertex $w$ and two new edges $v w$ and $v w^{\prime}$. A subdivision of $G$ is the graph obtained by a succession of elementary subdivisions.

We use the celebrated Kuratowski Graph Theorem. This theorem appears in [6]. The particular wording of the theorem is taken from [1]. In our work, we use it as a definition. 
Definition 114 A graph $G$ is planar if $G$ contains no subgraph isomorphic to a subdivision of $\mathcal{K}_{5}$ or $\mathcal{K}_{3,3}$.

First, we give a very simple lemma.

Lemma 115 Let $G$ be a connected graph. If $G$ has a connected subgraph $K$ such that there are at least four edges incident with $K$, then $\mathcal{C}(G)$ is not planar.

Proof. Let $\mathrm{e}_{1} \mathrm{e}_{2}, \mathrm{e}_{3}$ and $\mathrm{e}_{4}$ be the four edges incident with $K$ and $n$ be the size of $K$. If we define $L_{i}=K \cup \mathrm{e}_{i}$ for $i=1,2,3,4$, then we have four graphs with size $n+1$ that contain $K$ as a subgraph. This implies that $\left\langle K, L_{1}, L_{2}, L_{3}, L_{4}\right\rangle$ is a subgraph of $\mathcal{C}(G)$ isomorphic to $\mathcal{K}_{5}$. By Definition $114, \mathcal{C}(G)$ is not planar.

As an immediate consequence of this lemma, we have the following:

Remark 116 Let $G$ be a connected graph. If $G$ has a vertex of degree greater than 3 , then $\mathcal{C}(G)$ is not planar.

Actually having $\mathcal{C}(G)$ planar is pretty strong as the next lemma shows.

Lemma 117 Let $G$ be a connected graph with size greater than 3 . If $G$ has a vertex of degree three or more, then $\mathcal{C}(G)$ is not planar.

Proof. Let $v$ be that vertex, let $\mathrm{e}_{1}=v v_{1}, \mathrm{e}_{2}=v v_{2}$ and $\mathrm{e}_{3}=v v_{3}$ be the edges of $G$ all of them incident with the vertex $v$. Let $T=\left\langle\mathrm{e}_{1}, \mathrm{e}_{2}, \mathrm{e}_{3}\right\rangle$. Note that $T$ is the 3 -star; 
also, since $G$ is connected and has size greater than 3 , there is an edge $\mathrm{f}$ of $G$ incident with $T$. Without loss of generality, we can assume that $\mathrm{f}$ is incident with $v_{1}$.

Note that $\{v\},\left\langle e_{1}\right\rangle,\left\langle e_{2}\right\rangle$ and $\left\langle e_{3}\right\rangle$ are pairwise adjacent in $\mathcal{C}(G)$. This implies that $\mathcal{C}(G)$ contains an isomorphic copy of $\mathcal{K}_{4}$.

Let $K=\left\langle\mathrm{e}_{1}\right\rangle \cup\langle\mathbf{f}\rangle$. Note that $K$ is adjacent to $\left\langle\mathrm{e}_{1}\right\rangle$. We show three vertex disjoint paths joining $K$ with $\{v\},\left\langle\mathrm{e}_{2}\right\rangle$ and $\left\langle\mathrm{e}_{3}\right\rangle$.

The graph $K$ is adjacent to $\langle\mathbf{f}\rangle,\langle\mathbf{f}\rangle$ is adjacent to $\left\{v_{1}\right\}$ and $\left\{v_{1}\right\}$ is adjacent to $\{v\}$. This defines a path from $K$ to $\{v\}$. This path is illustrated in Figure 4.2(a).

Let $K_{2}=\left\langle\mathrm{e}_{1}\right\rangle \cup\left\langle\mathrm{e}_{2}\right\rangle$. The graph $K$ is adjacent to $K_{2}$ and $K_{2}$ is adjacent to $\left\langle\mathrm{e}_{2}\right\rangle$. This defines a path from $K$ to $\left\langle\mathrm{e}_{2}\right\rangle$. This path is illustrated in Figure 4.2(b).

Finally, let $K_{3}=\left\langle\mathrm{e}_{1}\right\rangle \cup\left\langle\mathrm{e}_{3}\right\rangle$. The graph $K$ is adjacent to $K_{3}$ and $K_{3}$ is adjacent to $\left\langle\mathrm{e}_{3}\right\rangle$. This defines a path from $K$ to $\left\langle\mathrm{e}_{3}\right\rangle$. This path is illustrated in Figure 4.2(c).

This implies that $\mathcal{C}(G)$ contains a subdivision of $\mathcal{K}_{5}$ and by Definition $114, \mathcal{C}(G)$ is not planar.

Theorem 118 Let $G$ be a connected graph. Then $\mathcal{C}(G)$ is planar if and only if $G$ is either a path, a cycle or the 3-star.

Proof. Note that the graphs $\mathcal{C}(G)$ of a path, a cycle and the 3 -star are planar (Figure 4.1). This shows the sufficiency part.

On the other hand, if every vertex is of degree at most 2 , then $G$ is either a path or a cycle.

Assume that there is a vertex of degree at least 3. Thus, $G$ is neither a cycle nor a path. 


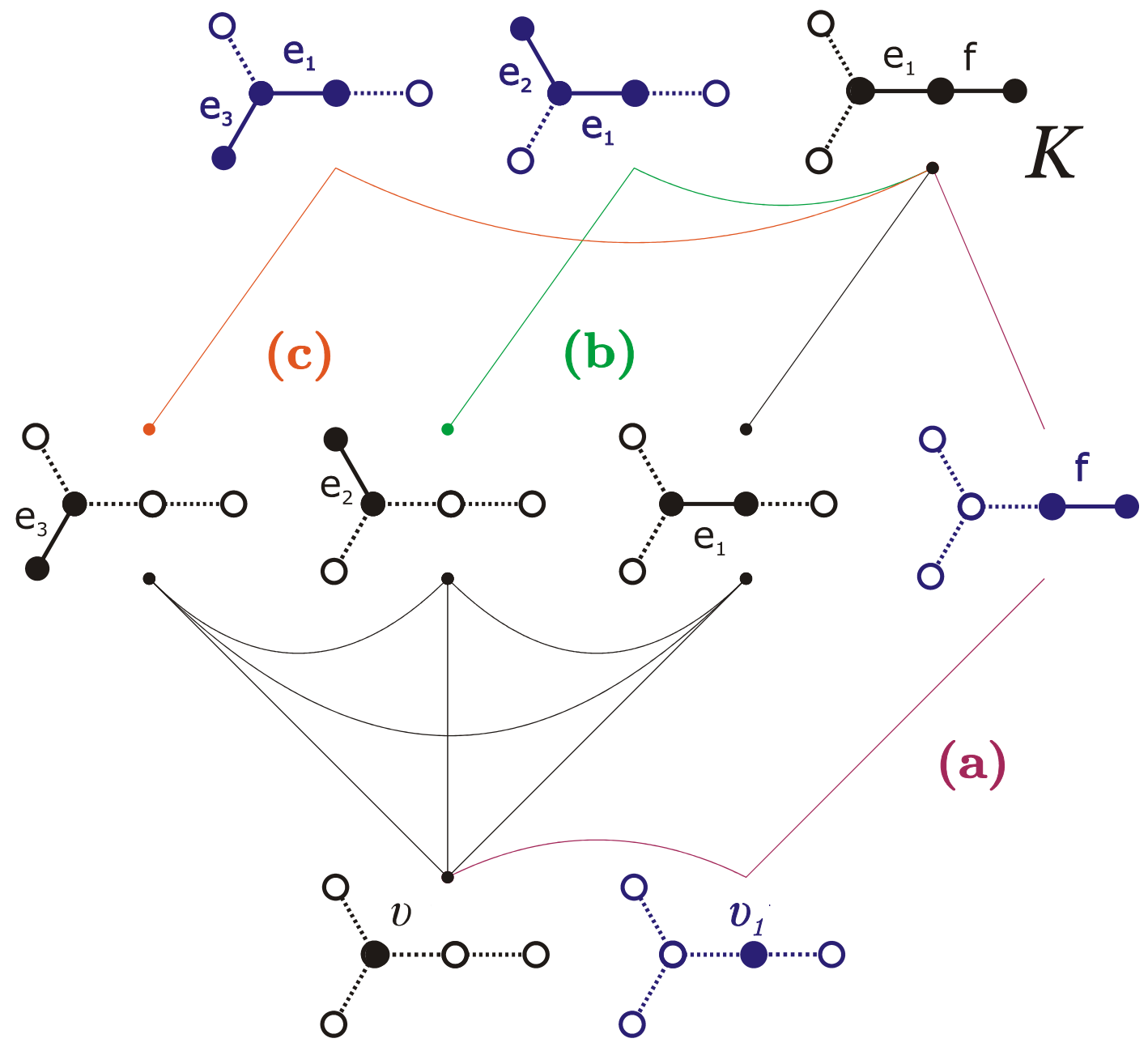

Figure 4.2: Proof of Lemma 117 
By Lemma 117, $G$ has size 3. Therefore, $G$ must be the 3 -star.

The hyperspace graphs of connected subgraphs of paths and cycles have very nice properties, the next two propositions show an example of those.

Proposition 119 The graph $\mathcal{C}(P)$ when $P$ is a path, is hamiltonian.

Proof. Let $P=v_{0} v_{1} \ldots v_{N}$. Consider $\mathcal{O}$ be the unique ordered path joining $\left\{v_{0}\right\}$ with $P$ in $\mathcal{C}(P)$. Define $P^{\prime}=v_{1} v_{2} \ldots v_{N}, \mathcal{P}_{0}=\left\{v_{o}\right\}\left\{v_{1}\right\} \ldots\left\{v_{N}\right\}, \mathcal{P}_{n}=\mathcal{Q}_{n}\left(P^{\prime}\right)$ for $n=$ $1,2, \ldots, N-1$ and $\mathcal{P}_{N}=\{P\}$. By Theorem $51, \mathcal{P}_{n}$ is a path for $n=1,2, \ldots, N-1$.

Now, for $n$ even, let $\mathrm{e}_{n}$ be the edge of $\mathcal{C}(P)$ joining the path of $P^{\prime}$ with length $n$ containing $v_{N}$, with the path of $P^{\prime}$ with length $n+1$ containing $v_{N}$.

Similarly for $n$ odd, define $\mathrm{e}_{n}$ to be the edge of $\mathcal{C}(P)$ joining the path of $P^{\prime}$ with length $n$ containing $v_{1}$, with the path of $P^{\prime}$ with length $n+1$ containing $v_{1}$.

So, $\mathcal{P}_{0} \mathrm{e}_{0} \mathcal{P}_{1} \mathrm{e}_{1} \ldots \mathcal{P}_{N-1} \mathrm{e}_{N-1} \mathcal{P}_{N} \mathcal{O}$ is a cycle in $\mathcal{C}(P)$. If $K$ is a subgraph of $P^{\prime}$ with size $n$, it is a vertex of $\mathcal{P}_{n}$ and if $K$ is not contained in $P^{\prime}$, then it is a vertex of $\mathcal{O}$. Therefore, $\mathcal{C}(P)$ is hamiltonian.

Proposition 120 The graph $\mathcal{C}(C)$ when $C$ is a cycle, is hamiltonian.

Proof. Let $v$ be any vertex in $C$. Consider $\mathcal{O}=O_{N} O_{N-1} \ldots O_{0}$ an order path with $O_{N}=C$ and $O_{0}=\{v\}$ in $\mathcal{C}(C)$. Define $\mathcal{P}_{0}=\mathcal{Q}_{0}(C)-\{\{v\}\}$ and let $H_{0}$ and $J_{0}$ be the endpoints of $\mathcal{P}_{0}$.

Inductively we define $H_{n}, J_{n}$ and $\mathcal{P}_{n}$ for $n<N$.

If $J_{n-1} \subset O_{n}$, then define $H_{n}$ to be the unique subgraph distinct from $O_{n}$ that contains $J_{n-1}$. 
If $J_{n-1} \nsubseteq O_{n}$, then define $H_{n}=j \operatorname{join}\left(O_{n-1}, J_{n-1}\right)$.

Observe that $J_{n-1} \subset H_{n}$. There is an edge $e_{n-1}$ of $\mathcal{C}(C)$ that joins $J_{n-1}$ with $H_{n}$.

Using Theorem 53, we have that $\mathcal{Q}_{n}$ is a cycle. Hence, $\mathcal{P}_{n}=\mathcal{Q}_{0}(C)-\left\{O_{n}\right\}$ is a path and $H_{n}$ is one endvertex of such path.

Let $J_{n}$ be the other endvertex of $\mathcal{P}_{n}$. Having defined $H_{n}, J_{n}$ and $\mathcal{P}_{n}$ for all $n$, let $\mathrm{e}_{N}=\mathrm{O}_{0} H_{0}$

Then $\mathcal{P}_{0} \mathrm{e}_{0} \mathcal{P}_{1} \mathrm{e}_{1} \ldots \mathcal{P}_{N-1} \mathrm{e}_{N-1} \mathcal{P}_{N} \mathcal{O} \mathrm{e}_{N}$ is a hamiltonian cycle of $\mathcal{C}(P)$.

The propositions above and Theorem 118 help us to characterize a path, a cycle and the 3-star.

Theorem 121 Let $G$ be a connected graph. Then $G$ is a path of size $N$ if and only if $C(G)$ is a planar hamiltonian graph having a vertex of degree 2 and with diameter $N$.

Proof. Let $G$ be a path with endvertices $v$ and $w$. Note that the diameter of $\mathcal{C}(G)$ is $N$. Since $G$ has exactly two connected subgraphs, $\operatorname{deg}_{\mathcal{C}(G)} G=2$. By Theorem 118 and Proposition $119, \mathcal{C}(G)$ is hamiltonian.

If $\mathcal{C}(G)$ is planar, then by Theorem $118, G$ has to be a path, a cycle or the 3 -star. Since $C(G)$ is a hamiltonian, $G$ cannot be the 3 -star. Since $\mathcal{C}(G)$ has a vertex of degree $2, G$ cannot be a cycle. So, $G$ is a path. However, the diameter of $\mathcal{C}(G)$ is $N$, then $G$ has size $N$.

Theorem 122 Let $G$ be a connected graph. Then $G$ is a cycle of size $N$ if and only if $C(G)$ is a planar hamiltonian graph such that every vertex has degree at least 3 and with diameter $N$. 
Proof. Assume that $G$ is a cycle of size $N$. Notice the following: the diameter of $\mathcal{C}(G)$ is $N$; all degenerate subgraphs have degree 4 in $\mathcal{C}(G)$; any proper nondegenerate subgraph of $G$ has degree 6 in $\mathcal{C}(G)$; the graph $G$, as a vertex of $\mathcal{C}(G)$, has degree $N$. By Theorem 118 and Proposition 119 he have that $\mathcal{C}(G)$ is hamiltonian.

Assume that $\mathcal{C}(G)$ is planar. By Theorem $118, G$ has to be a path, a cycle or the 3-star. Since $C(G)$ is a hamiltonian, $G$ cannot be the 3 -star. Since $\mathcal{C}(G)$ does not have a vertex of degree 2, $G$ cannot be a path. Then $G$ is a cycle. However, the diameter is $N$, then $G$ has size $N$.

Theorem 123 Let $G$ be a connected graph. Then $G$ is the 3 -star if and only if $C(G)$ is planar but not hamiltonian.

Proof. Let $G$ be the 3 -star with endvertices $v_{1}, v_{2}$ and $v_{3}$ and let $w$ be the interior vertex of $G$. Assume that $\mathcal{C}(G)$ is hamiltonian and $\mathcal{Y}$ is hamiltonian cycle. Since $\left\{v_{1}\right\}$ has degree 2 in $\mathcal{C}(G)$, the edge joining $\left\{v_{1}\right\}$ and $\{v\}$ has to be in $\mathcal{Y}$. However, the same happens for the edge joining $\left\{v_{2}\right\}$ and $\{v\}$ and the edge joining $\left\{v_{3}\right\}$ and $\{v\}$. Hence, $\{v\}$ has degree 3 in $\mathcal{Y}$. This is a contradiction, since $\mathcal{Y}$ is a cycle.

If $\mathcal{C}(G)$ is planar, then by Theorem $118, G$ has to be a path, a cycle or the 3 -star. But by Propositions 119 and 120, G has to be the 3-star.

\subsection{UNIQUENESS OF $\mathcal{C}(\mathbf{G})$.}

It will be nice to actually assure that any two distinct connected graphs have nonisomorphic hyperspace graphs. As we mention at the beginning of the chapter, the 
analogous result holds for the hyperspace of continua of topological graphs distinct from an arc and from a simple closed curve (see [2]).

First, we locate the vertex $G$ in the graph $\mathcal{C}(G)$. For that purpose, we locate the graphs with stitched neighborhoods in $\mathcal{C}(G)$.

Recall the definition of the neighborhood of a vertex in a graph (see Definition 77) and the definition of a stitched graph (see Definition 70).

Definition 124 If $K$ is a connected subgraph of $G$, we denote the neighborhood of $K$ in $\mathcal{C}(G)$ by $\mathcal{N}_{K}$

Define the following set:

$$
\mathcal{L}(G)=\left\{K \in \mathcal{C}(G): \mathcal{N}_{K} \text { is stitched }\right\}
$$

Note that if $G$ is a connected graph with size $N$, then $\mathcal{Q}_{N-1}(G)=\mathcal{N}_{G}$ and by Theorem 72 , we have the next remark.

Remark 125 Let $G$ be a connected graph. Then $G$ is not a cycle of size greater than 4 if and only if $G \in \mathcal{L}(G)$.

Now, let us find the other elements of $\mathcal{L}(G)$.

Lemma 126 Let $G$ be a connected graph and let $K$ be a connected non-empty subgraph of $G$. Then $K \in \mathcal{L}(G)$ if and only if one of the following statements hold:

(1) $K=G$ and $G$ is not a cycle of size greater than 4 . 
(2) $K=\{v\}$ for some endvertex $v$ of $G$

(3) $K=\{v\}$ for some vertex $v$ with degree 2 and contained in a cycle of size 3 .

Proof. Let $N$ be the size of $G$.

Note if (1) holds, then by Lemma 125 we have that $\mathcal{N}_{G}$ is stitched.

If $K$ is like in (2), then $\mathcal{N}_{K}$ is a single edge and therefore stitched

If $K$ is like in (3), then $\mathcal{N}_{K}$ is a cycle with size 4 and therefore stitched.

On the other hand, assume that $K$ is a proper nondegenerate and non-empty subgraph of $G$ with size $n$ such that $N_{K}$ is stitched.

Let $\mathcal{A}$ and $\mathcal{B}$ be a partition of $V\left(\mathcal{N}_{K}\right)$.

CLAIM 1. If $H \in V\left(\mathcal{Q}_{n-1}\right) \cap \mathcal{B}$, then $V\left(\mathcal{Q}_{n+1}\right) \cap V\left(\mathcal{N}_{K}\right) \subset \mathcal{B}$.

Assume $H \in \mathcal{B}$ and has size $n-1$. Since no subgraph of size $n+1$ is adjacent to $H$ in $\mathcal{C}(G)$, for every $L \in V\left(\mathcal{N}_{K}\right)$ with size $n+1, L \in \mathcal{B}$. Similarly, we can show the following claim:

CLAIM 2. If $L \in V\left(\mathcal{Q}_{n+1}\right) \cap \mathcal{B}$, then $V\left(\mathcal{Q}_{n-1}\right) \cap V\left(\mathcal{N}_{K}\right) \subset \mathcal{B}$.

Then using the claims above we can assume that $\mathcal{A} \subset V\left(\mathcal{Q}_{n}\right)$. Note that if $J \in \mathcal{A}$, then $K$ and $J$ are adjacent. Define $H=\operatorname{core}(J, K)$. Then $H \in \mathcal{B}$. Take $H^{\prime} \in \mathcal{B}-\{H\}$. Note that by Theorem $42, H^{\prime} \in \mathcal{B}$ is not adjacent to $J \in \mathcal{A}$. This contradicts the assumption that $\mathcal{N}_{G}$ is stitched. So, $H$ has to be degenerate.

Assume that for some vertex $v$ we have that $N_{\{v\}}$ has a spanning complete bipartite subgraph and that $\operatorname{deg}_{G} v \geq 2$.

If $\operatorname{deg}_{G} v \geq 3$, let $v_{1}, v_{2}$ and $v_{3}$ be three vertices adjacent with $v$. Without loss of generality, assume $v_{1} \in \mathcal{A}$. Then $\left\langle v v_{2}\right\rangle,\left\langle v v_{3}\right\rangle \in \mathcal{A}$ since $v_{1}$ is not adjacent to $\left\langle v v_{2}\right\rangle$ or $\left\langle v v_{3}\right\rangle$. 
Also, $v_{2}$ and $v_{3}$, are not adjacent to $\left\langle v v_{3}\right\rangle$. and $\left\langle v v_{2}\right\rangle$, respectively. Then $v_{2}, v_{3} \in \mathcal{A}$. Thus, $\left\langle v v_{1}\right\rangle \in \mathcal{A}$. However, $v_{1}, v_{2}$ and $v_{3}$ were any three vertices adjacent to $v$. So, $\mathcal{N}_{\{v\}}=\mathcal{A}$. This contradicts the fact that $\mathcal{B}$ is not empty. Then $\operatorname{deg}_{G} v=2$.

Now, we show that if for some vertex $v$ we have that $\operatorname{deg}_{G} v=2$ and $N_{\{v\}}$ is stitched with a partition $\mathcal{A}$ and $\mathcal{B}$, then (3) holds.

Let $v_{1}$ and $v_{2}$ be the only two vertices of $G$ that are adjacent to $v$. without loss of generality, assume $v_{1} \in \mathcal{A}$. Hence, $\left\langle v v_{2}\right\rangle \in \mathcal{A}$. Note that if $v_{2} \in \mathcal{A}$, then $\mathcal{N}_{\{v\}}=\mathcal{A}$ and this contradicts the fact that $\mathcal{B}$ is not empty. Hence, $v_{2} \in \mathcal{B}$. Since $N_{\{v\}}$ is stitched with partition $\mathcal{A}$ and $\mathcal{B}$ we have that in particular, $v_{1}$ has to be adjacent to $v_{2}$. So, $v v_{1} v_{2} v$ is a cycle of length 3. This finishes the proof.

Remark $127 G$ is a cycle of size greater than 4 if and only if $\mathcal{L}(G)=\varnothing$.

So, if we want to use the set $\mathcal{L}(G)$, then $G$ cannot be a cycle of size greater than 4.

Intuitively, it seems that $G$ is a different element from the rest of $\mathcal{L}(G)$. How different? The next lemma answers this question.

Lemma 128 Let $G$ be a connected graph such that $G$ is not a cycle of size greater than 4 . Then the following statements hold:

(1) If $|\mathcal{L}(G)|=2$ and $\{v\} \in \mathcal{L}(G)$ we have that $\operatorname{deg}_{\mathcal{C}(G)} G>\operatorname{deg}_{\mathcal{C}(G)}\{v\}$.

(2) Let $|\mathcal{L}(G)| \geq 3$ and let $N$ be the size of $G$. Then $K \in \mathcal{L}(G)$ is such that for every $L \in \mathcal{L}(G)-\{K\}, d(K, L)=N$ if and only if $K=G$. 
Proof. In order to prove (1), if $|\mathcal{L}(G)|=2$, then by Lemma 126, $\mathcal{L}(G)=\{\{v\}, G\}$ for some vertex $v$ of $G$.

If $v$ is an end-vertex of $G$, then $\operatorname{deg}_{\mathcal{C}(G)}\{v\}=2$. Since $G$ is not a path, $\operatorname{deg}_{\mathcal{C}(G)} G \geq$ 3. Therefore, statement (1) holds.

Assume that $v$ is a vertex of degree 2 and contained in a cycle of size 3 . So, $\operatorname{deg}_{\mathcal{C}(G)}\{v\}=4$. Then there are $v_{1}$ and $v_{2}$ vertices of $G$ that are adjacent to $v$. Note that the degrees of $v_{1}$ and $v_{2}$ must be greater than 2 (otherwise they are elements of $\mathcal{L}(G)$ ). Then $K=G-\{v\}$ is a connected subgraph with complementary edge set $\left\{v v_{1}, v v_{2}\right\}$. Note that the degrees of $v_{1}$ and $v_{2}$ in $G$ must be greater than 2 (otherwise they are elements of $\mathcal{L}(G))$. So, $v_{1}$ and $v_{2}$ are not endvertices of $K$ and this implies that $v_{1} v_{2}$ is not a terminal edge.

If $K$ contains a cycle, then there are two cycle edges e and $\mathrm{e}^{\prime}$ of $K$ distinct from $v_{1} v_{2}$. Therefore, e and $\mathrm{e}^{\prime}$ are cycle edges of $G$.

If $K$ is a tree, every endvertex of $K$ is an endvertex of $G$, since $v_{1}$ and $v_{2}$ are not endvertices. Then there are two terminal edges e and $\mathrm{e}^{\prime}$ of $K$ that are also terminal edges of $G$.

No matter which case we have that $v v_{1}, v v_{2}, v_{1} v_{2}$, e and $\mathrm{e}^{\prime}$ are removable edges of $G$. So, $\operatorname{deg}_{\mathcal{C}(G)} G \geq 5$. Therefore, $\operatorname{deg}_{\mathcal{C}(G)} G>\operatorname{deg}_{\mathcal{C}(G)}\{v\}$.

Now, we prove (2). By Lemma 126 and Remark 125, $\mathcal{L}(G)-\{G\} \subset \mathcal{Q}_{1}(G)$. For any $\{v\},\{w\} \in \mathcal{L}(G)-\{G\}$, since $G$ is not a path, we have that $d(\{v\},\{w\})=d(v, w)<N$. On the other hand, $d(G,\{v\})=N$ for every vertex $v$ of $G$. Therefore, if $K \in \mathcal{L}(G)$ is such that for every $L \in \mathcal{L}(G)-\{K\}$ we have that $d(K, L)=N$, then $K=G$. 
Now, that we have located the vertex $G$, the rest is somewhat easy.

Lemma 129 Let $G$ and $G^{\prime}$ be two connected graphs that are not cycles of size greater than 4. If there is an isomorphism $\phi: \mathcal{C}(G) \rightarrow \mathcal{C}\left(G^{\prime}\right)$, then $\phi(G)=\phi\left(G^{\prime}\right)$.

Proof. Note that $\phi(\mathcal{L}(G))=\mathcal{L}\left(G^{\prime}\right)$. Since $G$ and $G^{\prime}$ are not cycles of size greater than 4 , by Remark $125, G \in \mathcal{L}(G)$ and $G^{\prime} \in \mathcal{L}\left(G^{\prime}\right)$. Using the Lemma 128, one of the next statements holds:

(1) If $|\mathcal{L}(G)|=1$, then we have that $\phi(\{G\})=\left\{G^{\prime}\right\}$ and then $\phi(G)=G^{\prime}$.

(2) If $|\mathcal{L}(G)|=2$, then $\left|\mathcal{L}\left(G^{\prime}\right)\right|=2$. So, $\mathcal{L}(G)=\{G,\{v\}\}$ and $\mathcal{L}\left(G^{\prime}\right)=\left\{G^{\prime},\{w\}\right\}$. However, $\operatorname{deg}_{\mathcal{C}(G)} G>\operatorname{deg}_{\mathcal{C}(G)}\{v\}$ and $\operatorname{deg}_{\mathcal{C}\left(G^{\prime}\right)} G^{\prime}>\operatorname{deg}_{\mathcal{C}\left(G^{\prime}\right)}\{w\}$ therefore, $\phi(G)=\phi\left(G^{\prime}\right)$.

(3) If $|\mathcal{L}(G)| \geq 3$, then $G^{\prime}$ is the only element of $\mathcal{L}\left(G^{\prime}\right)$ satisfying that for every $L \in \mathcal{L}\left(G^{\prime}\right)-\{K\}$ we have that $d\left(G^{\prime}, L\right)=N$. Since $\phi$ is an isomorphism, $\phi(G)$ has also that property. Therefore, $\phi(G)=G^{\prime}$.

Finally, here is the theorem that we wanted to show.

Theorem 130 Let $G$ and $G^{\prime}$ be connected graphs. Then $G$ and $G^{\prime}$ are isomorphic if and only if $\mathcal{C}(G)$ and $\mathcal{C}\left(G^{\prime}\right)$ are isomorphic.

Proof. One implication is obvious by the construction of the hyperspace graph.

The case when one of the graphs is a path, a cycle or the 3 -star, by Theorem 118, $\mathcal{C}(G)$ is planar. This case is covered in Theorems 121, 122 and 123 in pages 62 to 63 in the previous section.

So, assume that neither $G$ nor $G^{\prime}$ are cycles and let $\phi: \mathcal{C}(G) \rightarrow \mathcal{C}\left(G^{\prime}\right)$ be an isomorphism. 
By Lemma 129, we have that $\phi(G)=G^{\prime}$. Consider

$$
N(G)=\max \{d(G, K): K \in V(\mathcal{C}(G))\}
$$

and

$$
\mathcal{R}(G)=\langle K \in V(\mathcal{C}(G)): d(G, K)=N\rangle .
$$

Since $\phi$ is an isomorphism between $\mathcal{C}(G)$ and $\mathcal{C}\left(G^{\prime}\right)$, we have that $\phi(\mathcal{R}(G))=$ $\mathcal{R}\left(G^{\prime}\right)$. Note that $\mathcal{Q}_{0}(G)=\mathcal{R}(G)$ and $\mathcal{Q}_{0}\left(G^{\prime}\right)=\mathcal{R}\left(G^{\prime}\right)$.

Then $\left.\phi\right|_{\mathcal{Q}_{0}(G)}: \mathcal{Q}_{0}(G) \rightarrow \mathcal{Q}_{0}\left(G^{\prime}\right)$ is an isomorphism. Therefore, $G$ and $G^{\prime}$ are isomorphic. 


\section{Chapter 5}

\section{RELATIONS BETWEEN $\mathcal{C}(\mathbf{G})$}

\section{AND $\mathbf{C}(\mathbf{X})$}

In Chapter 1, we discuss some similarities between hyperspaces of continua and hyperspace graphs. In this chapter we relate the two hyperspaces for topological trees. First, in Section 5.1, we give a geometric representation of $\mathcal{C}(G)$ in some euclidean space; thus, we can think of $\mathcal{C}(G)$ as a subset of a topological space. Then in Section 5.2, we generate a special set, called the induced set. Finally, in Section 5.3, we show that, for the case when the graph is a tree, the induced set is isomorphic to the hyperspace of subcontinua of the corresponding topological tree. 


\subsection{A PARTICULAR GEOMETRIC REPRESENTATION OF $\mathcal{C}(\mathbf{G})$.}

There is a particular geometric representation of the hyperspace graph of connected subgraphs. This representation is useful for relating the hyperspace graph to the hyperspace of subcontinua of the graph, when the graph is a topological tree. A similar process is done in [3] to describe the polyhedra that are homeomorphic to hyperspace of subcontinua of topological graphs.

Let $G$ be a connected graph with a vertex set $V(G)$ and edge set $E(G)$. Define $B=$ $\{\mathrm{e} \in E(G): \mathrm{e}$ is a bridge of $G\}$ and $C=\{\mathrm{e} \in E(G): \mathrm{e}$ is a cycle edge of $G\}$. Enumerate the edge set in the following way:

$$
E(G)=\left\{\mathrm{e}_{1}, \mathrm{e}_{2}, \ldots, \mathrm{e}_{P}, \mathrm{e}_{P+1}, \mathrm{e}_{P+2}, \ldots, \mathrm{e}_{P+R}\right\}
$$

such that

$$
B=\left\{\mathrm{e}_{1}, \mathrm{e}_{2}, \ldots, \mathrm{e}_{P}\right\} \text { and } C=\left\{\mathrm{e}_{P+1}, \mathrm{e}_{P+2}, \ldots, \mathrm{e}_{P+R}\right\}
$$

For any $i \in\{1,2, \ldots, P+2 R\}$, consider the function $\phi_{i}: V(\mathcal{C}(G)) \rightarrow \mathbb{R}$ as follows:

1. If $K$ is a nondegenerate subgraph of $G$ and $i \leq P$, then

$$
\phi_{i}(K)=\left\{\begin{array}{cl}
0 & \text { if } \mathrm{e}_{i} \notin E(K) \\
1 & \text { if } \mathrm{e}_{i} \in E(K)
\end{array}\right.
$$

2. If $K$ is a nondegenerate subgraph of $G$ and $P+1 \leq i \leq P+R$, then

$$
\phi_{i}(K)=\left\{\begin{array}{cc}
0 & \text { if } \mathrm{e}_{i} \notin E(K) \\
\frac{1}{2} & \text { if } \mathrm{e}_{i} \in E(K)
\end{array}\right.
$$


3. If $K=\{v\}$ and $i \leq P$, then $\phi_{i}(K)=\left\{\begin{array}{cc}0 & \text { if } \mathrm{e}_{i} \text { is incident with } v \\ -1 & \text { if } \mathrm{e}_{i} \text { is not incident with } v\end{array}\right.$

4. If $K=\{v\}$ and $P+1 \leq i \leq P+R$, then $\phi_{i}(K)=\left\{\begin{array}{cc}0 \quad & \text { if } \mathrm{e}_{i} \text { is incident with } v \\ -\frac{1}{2} & \text { if } \mathrm{e}_{i} \text { is not incident with } v\end{array}\right.$

5. If $P+R+1 \leq i \leq P+2 R$, then $\phi_{i}(K)=\phi_{i-R}(K)$

Remark 131 If $K$ is a nondegenerate subgraph of $G$, then the following statements hold:

- $\phi_{i}(K)=1$ if and only if $\mathrm{e}_{i} \in B \cap E(K)$.

- $\phi_{i}(K)=\phi_{i+R}(K)=\frac{1}{2}$ if and only if $\mathrm{e}_{i} \in C \cap E(K)$.

- $\phi_{i}(K)=0$ if and only if $\mathrm{e}_{i} \notin E(K)$.

- If $K$ has size $n$, then $\sum_{i=1}^{P+2 R} \phi_{i}(K)=n$.

- For any $J$ connected subgraph of $G$ such that $J$ is adjacent to $K, \phi_{i}(K) \neq \phi_{i}(J)$ if and only if $\mathrm{e}_{i} \in(E(J)-E(K)) \cup(E(K)-E(J))$.

Remark 132 Let $v$ and $w$ be two adjacent vertices of $G$ and let $\mathbf{e}_{i}$ be the edge between $v$ and $w$. Then $\phi_{j}(\{v\})=\phi_{j}(\{w\})$ if and only if $i=j$.

Now, define $\phi: \mathcal{C}(G) \rightarrow \mathbb{R}^{P+2 R}$ by

$$
\phi(K)=\left(\phi_{1}(K), \phi_{2}(K), \ldots, \phi_{P+2 R}(K)\right) .
$$


Note that $\phi$ is well defined; $\phi$ is also one-to-one by Remark 131. From now and on we denote $\phi(K)$ by $\vec{K}$.

Notation 133 For any $\vec{x}$ in $\mathbb{R}^{P+2 R}$, we denote the $i$-th coordinate of $\vec{x}$ by $x_{i}$.

Remark 134 Consider the hyperplane $\mathcal{P}_{n}=\left\{\vec{x} \in \mathbb{R}^{P+2 R}: \sum_{i=1}^{P+2 R} x_{i}=n\right\}$. Then $\phi\left(\mathcal{Q}_{n}\right) \subset$ $\mathcal{P}_{n}$ for $n \geq 1$. If $n=0$, then $\phi\left(\mathcal{Q}_{n}\right) \subset\left\{\vec{x} \in \mathbb{R}^{P+2 R}: \sum_{i=1}^{P+2 R} x_{i}<0\right\}$.

Using the function $\phi$, we construct a particular geometric representation of $\mathcal{C}(G)$ in $\mathbb{R}^{P+2 R}$. In order to do that, we define some line segments that join the images of adjacent vertices in $\mathcal{C}(G)$ under $\phi$.

For any pair of non-empty connected subgraphs $J$ and $K$ such that $J$ and $K$ are adjacent in $\mathcal{C}(G)$, define the following set in $\mathbb{R}^{P+2 R}$ :

$$
E_{J, K}=\left\{\vec{x} \in \mathbb{R}^{P+2 R}: \vec{x}=\vec{J}+t(\vec{K}-\vec{J})\right\}
$$

In other words, $E_{J, K}$ denotes the line segment joining $\vec{J}$ and $\vec{K}$ in $\mathbb{R}^{P+2 R}$. Also define

$$
\mathcal{R}(G)=\bigcup E_{J, K}
$$

Remark 135 If $J$ and $K$ are two nondegenerate level adjacent subgraphs of $G$, then $E_{J, K} \subset$ $\mathcal{P}_{n}$ 
Remark 136 Let $K$ be a connected subgraph of $G$ and let $J$ be a proper connected subgraph of $K$ with size $n$. Assume that $J$ and $K$ are adjacent in $\mathcal{C}(G)$.

(1) If $n \neq 0$, then $E_{J, K} \cap \mathcal{P}_{n}=\{J\}$ and $E_{J, K} \cap \mathcal{P}_{n+1}=\{K\}$.

(2) If $n=0$, then $E_{J, K} \cap \mathcal{P}_{1}=\{K\}$.

We have that the segments $E_{J, K}$ are contained in the hyperplanes $\mathcal{P}_{n}$ with $n \geq 1$, when the segment join images of nondegenerate level adjacent subgraphs $J$ and $K$ under $\phi$. If $E_{J, K}$ is as in Remark 135 and $E_{H, L}$ as in Remark 136, then $E_{J, K}$ and $E_{H, L}$ are either disjoint or meet in an endpoint.

Remark 137 Let $J$ and $K$ be two nondegenerate connected subgraphs of $G$ such that $J$ and $K$ are adjacent in $\mathcal{C}(G)$, let $\vec{x}$ be a point in $E_{J, K}$ such that it is not an endpoint of the line segment and let $x_{i}$ be the $i$-th coordinate of $\vec{x}$. Then we have the following:

(1) For $i \leq P, x_{i} \in(0,1)$ if and only if $\mathrm{e}_{i} \in(E(J)-E(K)) \cup(E(K)-E(J))$.

(2) For $P+1 \leq i \leq P+R, x_{i}=x_{i+R} \in\left(0, \frac{1}{2}\right)$ if and only if $\mathrm{e}_{i} \in(E(J)-E(K)) \cup$ $(E(K)-E(J))$

Remark 138 Let $\vec{x} \in E_{\{v\}, \mathrm{e}}$. Then $\vec{x}=\phi(\{v\})$ if and only if $x_{i} \geq 0$ for every $i \in$ $\{1,2, \ldots, P+2 R\}$

Remark 139 The following statements hold: 
(1) If $x \in E_{\{v\}, \mathrm{e}}$, then $\vec{x}=\phi(\{v\})$ if and only if for all $i \in\{1,2, \ldots, P+2 R\}$ we have that $x_{i} \leq 0$.

(2) $\mathrm{e}_{i}=v w$ if and only if for every $x \in E_{\{v\},\{w\}}$ we have that $x_{i}=0$.

Lemma 140 Let $v, w$ and $z$ be vertices and let $\mathrm{e}_{k}$ be an edge of $G$ such that $v$ is a vertex of $\mathrm{e}_{k}$ and $w$ is adjacent to $z$ in $G$. If the segments $E_{\{v\}, \mathbf{e}_{k}}$ and $E_{\{w\},\{z\}}$ intersect, then $v=w$ or $v=z$.

Proof. Let $\vec{x} \in E_{\{v\}, \mathbf{e}_{k}} \cap E_{\{w\},\{z\}}$ and let $\mathbf{e}_{j}$ be the edge of $G$ joining $w$ and $z$. Note that by Remark 139, we have that $x_{j}=0$ and $x_{i} \leq 0$ for $i \neq j$. Hence, $x_{i} \leq 0$ for all $i \in\{1,2, \ldots, P+2 R\}$ and since $\vec{x} \in E_{\{v\}, \mathbf{e}_{k}}$, using Remark 139, we have that $\vec{x}=\phi(v)$. Hence, $\phi_{j}(\{v\})=x_{j}=0$ and by the definition of $\phi_{j}, \mathbf{e}_{j}$ is incident with $v$. Therefore, $v=w$ or $v=z$.

Lemma 141 Let $v, w, y$ and $z$ be vertices of $G$ such that $v$ is adjacent to $w$ and $y$ is adjacent to $z$. If $\mathrm{e}_{j}$ is the edge joining $v$ and $w, \mathrm{e}_{k}$ is the edge joining $y$ and $z$, and the segments $E_{\{v\},\{w\}}$ and $E_{\{y\},\{z\}}$ intersect, then $\mathrm{e}_{j}=\mathrm{e}_{k}$ or $\mathrm{e}_{j}$ is adjacent to $\mathrm{e}_{k}$.

Proof. Let $x \in E_{\{v\},\{w\}} \cap E_{\{y\},\{z\}}$. Hence, by Remark 139, $x_{j}=0$ and $x_{k}=0$. Then using the definition of $E_{\{v\},\{w\}}, \vec{x}=\phi(\{v\})+t(\phi(\{w\})-\phi(\{v\}))$.

Thus, $(t-1) \phi_{k}(\{v\})=t \phi_{k}(\{w\})$.

If $t=1$, then $\phi_{k}(\{w\})=0$. This means that $e_{k}$ is incident with $w$.

If $t \neq 1$, then $\phi_{k}(\{v\})=\frac{t}{t-1} \phi_{k}(\{w\})$. However, $\phi_{k}(\{w\}) \leq 0$ and $t \in[0,1)$, so $\phi_{k}(\{v\}) \geq 0$. By the definition of $\phi_{k}$, we have that $\phi_{k}(\{v\})=0$ and therefore $e_{k}$ is incident to $v$. 
Note that the last two lemmas imply that two segments as in Lemma 140 or as in Lemma 141 can only intersect in a common endpoint.

So, we only need to show that the last conclusion holds also for two segments joining points in the same hyperplane $\mathcal{P}_{n}$ (for $n \geq 1$ ).

Remark 142 If $J$ and $K$ are nondegenerate level adjacent subgraphs of $G$, then for every $\vec{x} \in E_{J, K}$ we have that:

(1) If $\mathrm{e}_{i} \in \operatorname{core}(J, K)$, then $x_{i}=1$ for $i \in\{1,2, \ldots, P\}$ or $x_{i}=x_{i+R}=\frac{1}{2}$ for $i \in$ $\{P+1, P+2, \ldots, P+R\}$.

(2) If $\mathrm{e}_{i} \notin E(J) \cup E(K)$, then $x_{i}=0$ for all $i \in\{1,2, \ldots, P+R\}$ and, in the case that $i \in\{P+1, P+2, \ldots, P+R\}$, also $x_{i+R}=0$.

(3) If $\mathrm{e}_{i}$ is a complementary edge of core $(J, K)$ in $J$ (or in $K$ ) and $\vec{x}$ is not an endpoint in $E_{J, K}$, then $x_{i} \in(0,1)$ for $i \in\{1,2, \ldots, P\}$ and $x_{i}=x_{i+R} \in\left(0, \frac{1}{2}\right)$ for $i \in\{P+1, P+2, \ldots, P+R\}$.

Lemma 143 Let $H, J, K$ and $L$ be nondegenerate connected subgraphs with the same size such that $J$ and $K$ are level adjacent, $H$ and $L$ are level adjacent. If the segments $E_{J, K}$ and $E_{H, L}$ are distinct and they intersect, then they intersect in a common endpoint.

Proof. Let $\vec{x} \in E_{J, K} \cap E_{H, L}$, let $e_{j}$ and $e_{k}$ be the complementary edges of core $(J, K)$ in $J$ and $K$ respectively.Assume that $\vec{x}$ is not and endpoint of $E_{J, K}$. So, 
$x_{j} \in(0,1)$ if $j \leq P$ or $x_{j} \in\left(0, \frac{1}{2}\right)$ if $P+1 \leq j \leq P+2 R$. This implies that $\vec{x} \neq \vec{H}$ and $\vec{x} \neq \vec{L}$. So, $\vec{x}$ is not an endpoint of $E_{H, L}$.

Now, $\mathrm{e}_{i} \in \operatorname{core}(J, K)$ if and only if $x_{i}=1$ if $j \leq P$ or $x_{i}=\frac{1}{2}$ if $P+1 \leq j \leq P+2 R$, by Remark 142 , it is equivalent to say that $\mathrm{e}_{i} \in \operatorname{core}(H, L)$. Therefore, $\operatorname{core}(J, K)=$ core $(H, L)$. By a similar argument, it can be shown that $E(J) \cup E(K)=E(H) \cup E(L)$. So, either $H=\operatorname{core}(H, L) \cup \mathrm{e}_{j}$ or $H=\operatorname{core}(H, L) \cup \mathrm{e}_{k}$. Then $\vec{H}=\vec{J}$ or $\vec{H}=\vec{K}$. This implies that $E_{J, K}=E_{H, L}$ and this is a contradiction. Therefore, we have that $\vec{x}$ has to be an endpoint of both $E_{J, K}$ and $E_{H, L}$.

So, we have shown that if a pair of segments $E_{J, K}$ are distinct and they intersect, then they intersect in a common endpoint. This leads to the following:

Theorem $144 \mathcal{R}(G)$ is a geometric representation of $\mathcal{C}(G)$ in $\mathbb{R}^{P+2 R}$.

\subsection{INDUCED SET OF $\mathcal{C}(\mathbf{G})$.}

In this section we assume that all our graphs are trees. We continue using the enumeration and notation defined in the previous section. Immediately, we can say the following:

Remark 145 If $G$ is a tree with size $N$, then $E(G)=B=\left\{\mathrm{e}_{1}, \mathrm{e}_{2}, \ldots, \mathrm{e}_{N}\right\}$ and $\mathcal{R}(G)$ is a set of $\mathbb{R}^{N}$. 
First, we define a particular subset of $\mathbb{R}^{N}$ based on $\mathcal{R}(G)$. This set is called the induced set of $\mathcal{C}(G)$.

Let $\left\{\vec{z}_{1}, \vec{z}_{2}, \ldots, \vec{z}_{N}\right\}$ be the canonical base of $\mathbb{R}^{N}$. Observe that $\vec{z}_{i}=\phi\left(\left\langle\mathrm{e}_{i}\right\rangle\right)$ for $i \in\{1,2, \ldots, N\}$

Define also the set $I(K)=\left\{i \in\{1,2, \ldots, P\}: \mathrm{e}_{i}\right.$ is incident with $\left.K\right\}$

For every edge $\mathrm{e}_{i}$ of $G$ joining the vertices $v$ and $w$, consider the subset $T_{i}$ of $\mathbb{R}^{N}$ that is the convex hull of the points $\vec{v}, \vec{w}$ and $\vec{z}_{i}$ in $\mathbb{R}^{N}$; that is,

$$
T_{i}=\left\{\vec{x} \in \mathbb{R}^{N}: \vec{x}=t_{1} \vec{v}+t_{2} \vec{w}+t_{3} \vec{z}_{i} \text { where } t_{1}+t_{2}+t_{3}=1\right\}
$$

For a given vertex $v$ of $G$, we define the following two sets (both sets are illustrated in Figure 5.1):

The set $S_{v}$ is the convex hull of the point $\vec{v}$ and the points $\vec{z}_{i}$ in $\mathbb{R}^{N}$ where $\mathrm{e}_{i}$ is incident with $v$,

$$
S_{v}=\left\{\vec{x} \in \mathbb{R}^{N}: \vec{x}=t v+\sum_{i \in I(\{v\}\}} t_{i} z_{i} \text { with } t+\sum_{i \in I(\{v\}\}} t_{i}=1\right\}
$$

The set $R_{v}$ is a truncated cell in $\mathbb{R}^{N}$,

$$
R_{v}=\left\{\vec{x} \in \mathbb{R}^{N}: \vec{x}=\sum_{i \in I(\{v\}\}} t_{i} \vec{z}_{i} \text { with } t_{i} \geq 0 \text { and } 1 \leq \sum_{i \in I(\{v\}\}} t_{i} \leq \operatorname{deg}(v)\right\}
$$


Note that

$$
S_{v} \cap R_{v}=\left\{\vec{x} \in \mathbb{R}^{N}: \vec{x}=\sum_{\mathrm{e}_{i} \in I(\{v\})} t_{i} \vec{z}_{i} \text { with } t_{i} \geq 0 \text { and } \sum_{i \in I(K)} t_{i}=1\right\}
$$

Finally, define

$$
M_{v}=S_{v} \cup R_{v} .
$$

It is easy to check that $M_{v}$ is homeomorphic to a $k$-cell, where $k$ is the degree of

$v$.
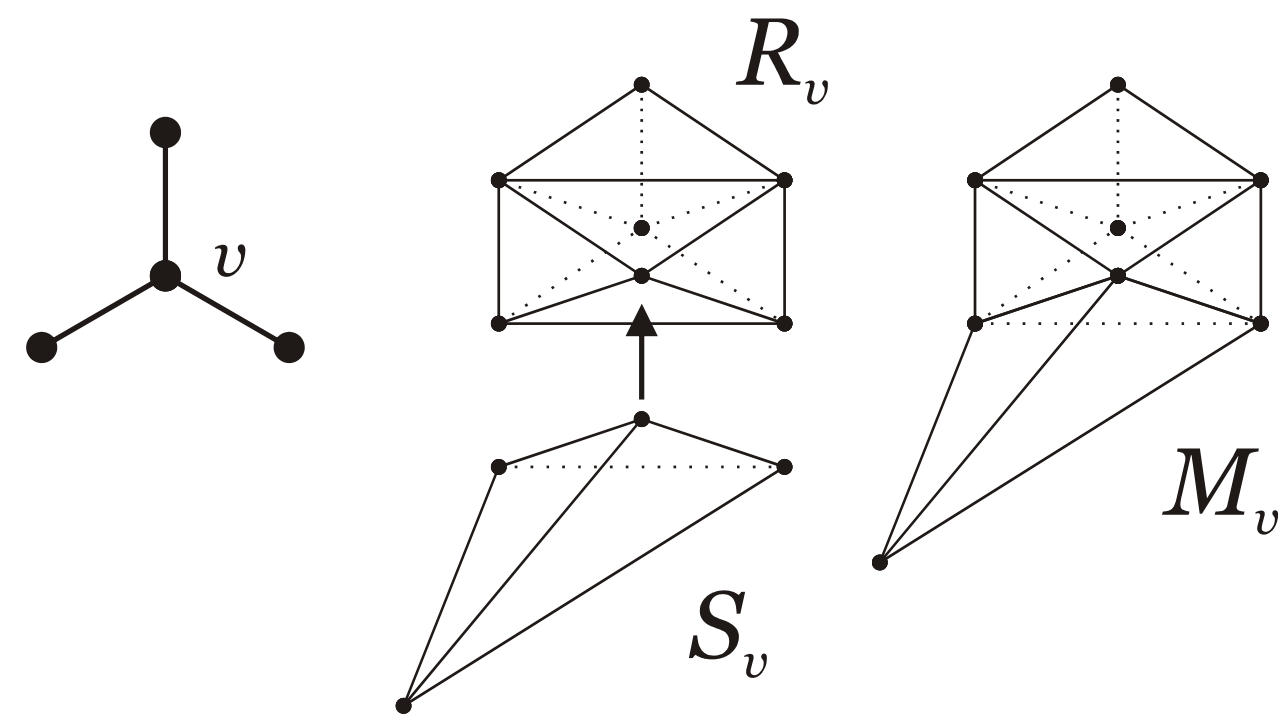

Figure 5.1: The sets $R_{v}, S_{v}$ and $M_{v}$ when $v$ is a degree 3 vertex. 
For every nondegenerate connected subgraph $K$ of $G$, define the set $M_{K}$ by

$$
M_{K}=\left\{\vec{x} \in \mathbb{R}^{N}: \vec{x}=\vec{K}+\sum_{i \in I(\{v\}\}} t_{i} \vec{z}_{i} \text { with } t_{i} \in[0,1]\right\}
$$

Statements in the next two remarks are easy consequences of the definition of $M_{K}$ :

Remark 146 If $|E(K, G)|=l$, then the set $M_{K}$ is homeomorphic to a l-cell.

Remark 147 Let $\vec{x} \in M_{K}$. Then:

1. If $\mathrm{e}_{i} \in E(K)$, then $x_{i}=1$.

2. If $\mathrm{e}_{i} \in E(K, G)$, then $x_{i} \in[0,1]$.

3. If $\mathrm{e}_{i} \notin E(K) \cup E(G, K)$, then $x_{i}=0$.

Lemma 148 Let $K$ and $K^{\prime}$ be two distinct connected nondegenerate subgraphs of $G$. If $M_{K} \cap M_{K^{\prime}} \neq \varnothing$, then $V(K) \cap V\left(K^{\prime}\right) \neq \varnothing$.

Proof. Let $\vec{x} \in M_{K} \cap M_{K^{\prime}}$. Since $K$ and $K^{\prime}$ are different subgraphs of $G$, we can assume (without loss of generality) that there is $\mathrm{e}_{i} \in E\left(K^{\prime}\right)-E(K)$. Then we have that $x_{i}=1$. By Remark 147, the edge $\mathrm{e}_{i} \in E(K) \cup E(G, K)$. However $\mathrm{e}_{i} \notin E(K)$, hence $\mathrm{e}_{i} \in E(G, K)$. There is a vertex $v$ of $\mathrm{e}_{i}$ that is also a vertex of $K$. If $\mathrm{e}_{i} \in E\left(K^{\prime}\right)$, then both vertices of $\mathrm{e}_{i}$ ( $v$ in particular) are vertices of $K^{\prime}$. Therefore, $v \in V(K) \cap V\left(K^{\prime}\right)$.

Lemma 149 Let $K$ and $K^{\prime}$ be two distinct connected nondegenerate subgraphs of $G$. Then $M_{K} \cap M_{K^{\prime}} \neq \varnothing$ if and only if $K \subset N\left(1, K^{\prime}\right)$ and $K^{\prime} \subset N(1, K)$. 
Proof. Let $\vec{x} \in M_{K} \cap M_{K^{\prime}}$ and assume that $K \not \subset N\left(1, K^{\prime}\right)$. Then there is an edge $\mathrm{e}_{i}$ of $K$ that is not in $E\left(K^{\prime}\right) \cup E\left(G, K^{\prime}\right)$. Hence, $x_{i}=0$. This contradicts Remark 147. This shows one implication.

For the remaining implication, just note that $K \cup K^{\prime}$ is a connected subgraph of $G$; since $K \subset N\left(1, K^{\prime}\right)$ and $K^{\prime} \subset N(1, K)$, we have that $\overrightarrow{K \cup K^{\prime}} \in M_{K} \cap M_{K^{\prime}}$.

Let $G$ be a connected non-empty graph and

$$
\mathcal{P}(G)=\left(\bigcup_{K \in V(\mathcal{C}(G))} M_{K}\right) \cup\left(\bigcup_{i=1}^{N} T_{i}\right)
$$

Definition 150 The set $\mathcal{P}(G)$ is called the induced set of $\mathcal{C}(G)$.

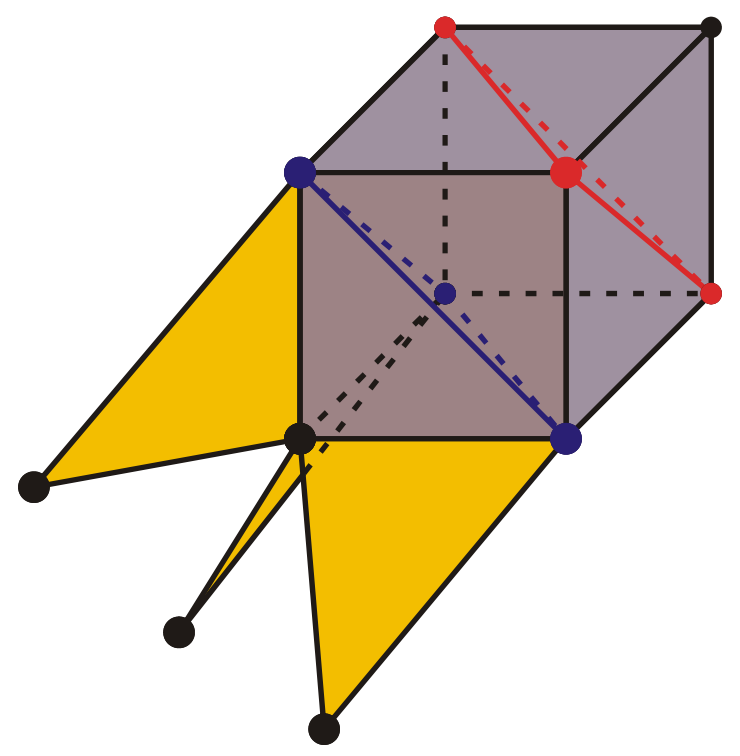

Figure 5.2: The induced set of the 3 -star 


\subsection{RELATIONS BETWEEN $\mathcal{C}(\mathrm{G})$ AND C $(\mathrm{X})$ FOR TREES.}

Definition 151 A compact, connected non-empty metric space is a continuum A subcontinuum is a subset that is a continuum.

Consider a topological tree $X$. This means that $X$ can be written as a finite union

of $\operatorname{arcs} A_{i}$, any two of which intersect in at most one endpoint and that $X$ does not contain a simple closed curve. Let $W=\left\{v \in X: v\right.$ is an endpoint of an $\left.\operatorname{arc} A_{i}\right\}$.

Remark 152 By adding some points to $W$, we can create a finite set $V$ in $X$ such that $X$ is a finite union of arcs $E_{i}$ such that only intersect at exactly one of their endpoints and the set of endpoints of the $E_{i}$ is $V$. Let $E=\left\{E_{1}, E_{2}, \ldots, E_{N}\right\}$ be the set of such arcs.

Let us define a metric for $X$.

Note that every arc $E_{i}$ is homeomorphic to $[0,1]$. This induces a metric in each $E_{i}$. Now, for every $\operatorname{arc} A$ in $X$, define length $(A)=\sum$ length $\left(A \cap E_{i}\right)$. Finally, we define the distance between two points in $X$ by the shortest length of the arcs joining those two points.

Next, consider the abstract graph $G_{X}$ defined by:

$V\left(G_{X}\right)=V$ and $E\left(G_{X}\right)=\left\{v w: v\right.$ and $w$ are endpoints of $E_{i}$ for some $\left.i\right\}$.

Note that we can denote $E\left(G_{X}\right)$ simply by $E$, since every edge in $E\left(G_{X}\right)$ can be represented by an element of $E$. 
We define $C(X)$ and show that $\mathcal{P}\left(G_{X}\right)$ is homeomorphic to $C(X)$. Observe that every connected subgraph $K$ of $G_{X}$ can be seen as a subcontinua of $X$.

For any subcontinuum $A$ of $X$ and any $\varepsilon>0$, define:

$$
N_{\varepsilon}(A)=\{x \in X: \text { there is } a \in A \text { such that } d(x, a) \leq \varepsilon\} .
$$

Definition 153 Let $X$ be a continuum with a metric d. For any two subcontinua $A$ and $B$ of $X$, the Hausdorff metric induced by $d$ is defined by:

$$
H_{d}(A, B)=\inf \left\{\varepsilon>0: A \subset N_{\varepsilon}(B) \text { and } B \subset N_{\varepsilon}(A)\right\} .
$$

Definition 154 Let $X$ be a continuum. The hyperspace of subcontinua of $X$ is the set

$$
C(X)=\{A \subset X: A \text { is a subcontinuum of } X\}
$$

with the topology induced by the Hausdorff metric.

Proposition 155 If $K$ is a connected subgraph of $X$, then we have that

$$
N_{1}(K)=K \cup\left(\bigcup_{i \in I(K)} E_{i}\right) .
$$

Proof. Let $x \in N_{1}(K)$. If $x \in K$, we are done; so, let us assume that $x \notin K$. There is $k \in K \cap V$ such that $d(x, k) \leq 1$. Since $k \in V, k$ and $x$ are contained in the same $\operatorname{arc} E_{i} \in E$. Then, $k \in E_{i} \cap K$. Thus, $i \in I(K)$.

On the other hand if $x \in E_{i}$ and $i \in I(K)$, then there is a $k \in K \cap V$ such that $k$ is an endpoint of $E_{i}$. So, $d(x, k) \leq 1$ and therefore $x \in N_{1}(K)$.

For any connected subgraph $K$ of $G_{X}$, consider the following subset of $C(X)$ : 


$$
\mathcal{M}_{K}=\left\{A \in C(X): K \subset A \subset N_{1}(A)\right\}
$$

This kind of set was first defined by R. Duda in [2]. Note that, if $A \in \mathcal{M}_{K}$ and $E_{i}$ an element of $E$ such that $E_{i}$ is not contained in $K$ but intersects $K$, then $A \cap E_{i} \neq \varnothing$, since $K \subset A$.

We use these sets to define a homeomorphism between $C(X)$ and $\mathcal{P}\left(G_{X}\right)$. Since $X$ is a tree, we have the next remark:

Remark 156 Let $K$ be a connected subgraph of $X$ and let $A \in \mathcal{M}_{K}$. If $E_{i}$ is an element of $E$ such that $E_{i}$ is incident with $K$, then $E_{i} \cap A$ is a subarc of $E_{i}$.

Now, we define an auxiliary function.

Let $K$ be subgraph of $G_{X}$ and let $i \in I(K)$. For any $A \in \mathcal{M}_{K}$, by Lemma 156 , $A \cap E_{i}$ is an arc. Define $h_{i}: \mathcal{M}_{K} \rightarrow[0,1]$ by $h_{i}(A)=\operatorname{lenght}\left(A \cap E_{i}\right)$. Note that the function $h_{i}$ is continuous.

For any $K$ connected nondegenerate subgraph of $G_{X}$, we can define a function $h_{K}: \mathcal{M}_{K} \rightarrow M_{K}$ by:

$$
h_{K}(A)=\vec{K}+\sum_{i \in I(K)} h_{i}(K) \vec{z}_{i}
$$

For any degenerate connected subgraph $K=\{v\}$ of $G_{X}$, we define a function $h_{v}: \mathcal{M}_{\{v\}} \rightarrow M_{v}$ by: 


$$
h_{v}(A)=\left\{\begin{array}{cc}
\overrightarrow{\{v\}}+\sum_{i \in I(\{v\})} h_{i}(A)\left(\vec{z}_{i}-\overrightarrow{\{v\}}\right) & \text { if } \sum_{i \in I(\{v\})} \text { length }\left(A \cap E_{i}\right) \leq 1 \\
\sum_{i \in I(\{v\})} h_{i}(A) \vec{z}_{i} & \text { if } \sum_{i \in I(\{v\})} \text { length }\left(A \cap E_{i}\right) \leq 1
\end{array}\right.
$$

Lemma 157 The function $h_{K}$ is a homeomorphism for every connected nondegenerate subgraph $K$ of $G_{X}$.

Proof. Since the definition of the functions $h_{i}$, it is clear that for any $A \in \mathcal{M}_{K}$ we have that $h_{K}(A) \in M_{K}$. Also, since all the functions $h_{i}$ are continuous, $h_{K}$ is continuous.

Let $A$ and $A^{\prime}$ be two different elements of $\mathcal{M}_{K}$. Without loss of generality, assume that $A-A^{\prime} \neq \varnothing$ and let $x \in A-A^{\prime}$. Since $K$ is contained in both $A$ and $A^{\prime}, x \in E_{i}$ for some $\operatorname{arc} E_{i}$ outside of $K$. We have that $E_{i}$ intersects $K$ in exactly one point $v$. Then $A \cap E_{i}$ and $A^{\prime} \cap E_{i}$ are two distinct subarcs of $E_{i}$ containing $v$. This implies that $A^{\prime} \cap E_{i} \varsubsetneqq A \cap E_{i}$. Thus, $h_{i}\left(A^{\prime}\right)<h_{i}(A)$. Therefore, $h_{K}$ is a one-to-one function.

Finally, we show that $h_{K}$ is a surjection.

Let $\vec{x} \in M_{K}$. For every $i \in I(K)$ we have that $E_{i}$ is an arc outside of $K$ that intersects $K$ in exactly one endpoint $v$ of $E_{i}$. Define the finite collection of $\operatorname{arcs}\left\{A_{i}\right\}_{i \in I(K)}$ such that $A_{i}$ is the subarc of $E_{i}$ containing $v$ and of length $x_{i}$.

Define $A=K \cup\left(\bigcup_{i \in I(K)} A_{i}\right)$. Note that $A$ is a subcontinuum of $X$ and $A \in \mathcal{M}_{K}$ since the length of the $\operatorname{arcs} A_{i}$ is less or equal to 1 . By the way we constructed $A$, it is easy to check that $h(A)=\vec{x}$.

We can conclude that $h_{K}$ is a surjection and, therefore, that $h_{K}$ is a homeomorphism. 
Lemma 158 The function $h_{v}$ is a homeomorphism.

Proof. First, we show that the function $h_{v}$ is well defined.

$$
\begin{aligned}
& \text { Assume that } A \text { is such that } \sum_{i \in I(\{v\})} \operatorname{length}\left(A \cap E_{i}\right)=1 \text {. Then, } \\
& \overrightarrow{\{v\}}+\sum_{i \in I(\{v\})} h_{i}(A)\left(\overrightarrow{\left\langle\mathrm{e}_{i}\right\rangle}-\overrightarrow{\{v\}}\right)= \\
& \left(1-\sum_{i \in I(\{v\})} h_{i}(A)\right) \overrightarrow{\{v\}}+\sum_{i \in I(\{v\})} h_{i}(A) \overrightarrow{\left\langle\mathrm{e}_{i}\right\rangle}=\sum_{i \in I(\{v\})} h_{i}(A) \overrightarrow{\left\langle\mathrm{e}_{i}\right\rangle} .
\end{aligned}
$$

So, $h_{v}$ is well defined and continuous.

By a similar argument as in the proof of Lemma 157, it can be shown that $h_{v}$ is a one-to-one surjection and, therefore, is a homeomorphism.

In order to give a homeomorphism between $C(X)$ and $\mathcal{P}\left(G_{X}\right)$ we need to define some homeomorphisms $g_{i}$ between $C\left(E_{i}\right)$ and $T_{i}$.

Take $E_{i}$ one of the arcs defined at the beginning of this section. Let $v$ and $w$ be the endpoints of $E_{i}$.

Let $A$ be a subcontinuum of $E_{i}$.

If $A=\{a\}$ define $b=a$.

If $A$ is nondegenerate, then $A$ is a subarc of $E_{i}$. Let $a$ and $b$ be the endpoints of $A$; assume, without loss of generality, that $a$ is the endpoint of $A$ closest to $v$ in $E_{i}$, and, $b$ the endpoint of $A$ closest to $w$ in $E_{i}$.

Define $f_{i}: C\left(E_{i}\right) \rightarrow T_{i}$ by follows:

$$
f_{i}(A)=d(b, w) \overrightarrow{\{v\}}+d(a, v) \overrightarrow{\{w\}}+[1-d(a, v)-d(b, w)] \overrightarrow{\left\langle\mathrm{e}_{i}\right\rangle}
$$

Note that $f_{i}$ is a homeomorphism. 
Finally, we can define a homeomorphism between $C(X)$ and $\mathcal{P}\left(G_{X}\right)$. Define $h$ : $C(X) \rightarrow \mathcal{P}\left(G_{X}\right)$ by:

$$
h(A)=\left\{\begin{array}{cc}
f_{i}(A) & \text { if } A \in C\left(E_{i}\right) . \\
h_{v}(A) & \text { if } A \in \mathcal{M}_{v} \\
h_{K}(A) & \text { if } A \in \mathcal{M}_{K} .
\end{array}\right.
$$

Theorem 159 The function $h$ is a homeomorphism.

Proof. Note that it is sufficient to prove that $h$ is well defined (since $f_{i}, h_{v}$ and $h_{K}$ are known to be homeomorphisms).

(1) Observe that if $C\left(E_{i}\right) \cap C\left(E_{j}\right) \neq \varnothing$, then $E_{i}$ and $E_{j}$ intersect. By the way those arcs were taken, $E_{i}$ and $E_{j}$ intersect only in one common endpoint $v$. Hence, $C\left(E_{i}\right) \cap$ $C\left(E_{j}\right)=\{\{v\}\}$. By the definition of $f_{i}$ and $f_{j}$, we have that $f_{i}(\{v\})=\overrightarrow{\{v\}}=f_{j}(\{v\})$.

(2) Let $A \in C\left(E_{i}\right) \cap \mathcal{M}_{v}$. Then $A$ is subarc of $E_{i}$ such that $v$ is the common endpoint of $A$ and $E_{i}$. Let $b$ be the other endpoint of $A$ and let $w$ be the other endpoint of $E_{i}$. By the definition of $f_{i}$ we have that $f_{i}(A)=d(b, w) \overrightarrow{\{v\}}+[1-d(b, w)] \overrightarrow{\left\langle\mathbf{e}_{i}\right\rangle}$. Now, using the definition of $h_{v}$, we have that

$$
\begin{aligned}
h_{v}(A)= & \overrightarrow{\{v\}}+\text { length }(A)\left[\overrightarrow{\left\langle\mathrm{e}_{i}\right\rangle}-\overrightarrow{\{v\}}\right]=\overrightarrow{\{v\}}+d(v, b)\left[\overrightarrow{\left\langle\mathrm{e}_{i}\right\rangle}-\overrightarrow{\{v\}}\right] \\
& =[1-d(v, b)] \overrightarrow{\{v\}}+d(v, b) \overrightarrow{\left\langle\mathbf{e}_{i}\right\rangle}=d(b, w) \overrightarrow{\{v\}}+[1-d(b, w)] \overrightarrow{\left\langle\mathrm{e}_{i}\right\rangle}
\end{aligned}
$$

(3) Note that $C\left(E_{i}\right) \cap \mathcal{M}_{K}=\varnothing$ for subgraphs $K$ containing more than one arc. So, $C\left(E_{i}\right) \cap K \neq \varnothing$ if and only if $K=E_{i}$. Hence, $C\left(E_{i}\right) \cap \mathcal{M}_{K}=\left\{E_{i}\right\}$. Thus, $f_{i}\left(E_{i}\right)=$ $\overrightarrow{\left\langle\mathrm{e}_{i}\right\rangle}=h_{K}\left(E_{i}\right)$. 
(4) Let $A \in \mathcal{M}_{v} \cap \mathcal{M}_{w}$. Note that for every $p \in X-E_{i}$, the point $p$ is in an $\operatorname{arc} E_{j}$ different from $E_{i}$. Either $d(p, v)>1$ or $d(p, w)>1$. Then $A=E_{i}$. Thus, $h_{v}\left(E_{i}\right)=h\left(E_{i}\right)=\overrightarrow{\left\langle\mathrm{e}_{i}\right\rangle}$.

(5) Let $A \in \mathcal{M}_{v} \cap \mathcal{M}_{K}$ with $K$ a nondegenerate subgraph. So, $K=\bigcup_{k=1}^{m} E_{i_{k}}$ with $E_{i_{k}}$ having $v$ as an endpoint for all $k$.

$$
\text { So, } \begin{aligned}
h_{v}(A) & =\sum_{e_{i} \in E(K)} \overrightarrow{\left\langle\mathrm{e}_{i}\right\rangle}+\sum_{e_{i} \in E(G,\{v\})-E(K)} h_{i}(A) \overrightarrow{\left\langle\mathrm{e}_{i}\right\rangle} \\
& =\vec{K}+\sum_{e_{i} \in I(K) \cap I(\{v\})} h_{i}(A) \overrightarrow{\left\langle\mathrm{e}_{i}\right\rangle}=h_{K}(A) .
\end{aligned}
$$

(6) Let $A \in \mathcal{M}_{K} \cap \mathcal{M}_{K^{\prime}}$, let $\vec{x}=h_{K}(A)$, let $\vec{y}=h_{K^{\prime}}(A)$ and let $i \in\{1,2, \ldots, N\}$. We denote $h_{i}$ to be the auxiliary function defining $h_{K}$ and $h_{i}^{\prime}$ the one for $h_{K^{\prime}}$. If $i \notin I(K) \cup I\left(K^{\prime}\right)$, we have that $x_{i}=y_{i}=0$. Similarly, if $E_{i} \subset K \cap K^{\prime}$, we have that $x_{i}=y_{i}=1$. Assume that $i \notin I(K) \cap I\left(K^{\prime}\right)$. Without loss of generality, let $i \notin I(K)$. This implies that $E_{i} \not \subset N_{1}(A)$. However, $A \in \mathcal{M}_{K}$. Then $A \subset N_{i}(K)$. Hence, $A \cap E_{i}$ is degenerate. This implies that $h_{i}(A)=0$. Thus, $x_{i}=0$. We have also that $y_{i}=0$.

If $i \in I(K) \cap I\left(K^{\prime}\right)$, then $h_{i}(A)=h_{i}^{\prime}(A)$ and $x_{i}=y_{i}$. Therefore, $h_{K}(A)=h_{K^{\prime}}(A)$. This finishes the proof. 


\section{Chapter 6}

\section{QUESTIONS}

In this last chapter we state questions for further research and study. We include comments with some of the questions.

\subsection{QUESTIONS ABOUT THE SIZE LEVELS}

1. Characterize the graphs that are size levels.

2. Given a connected graph, can we find a formula to compute the number of vertices of each size level?

The case of the 1-st size level is trivial.

3. Given a connected graph, can we find a formula to compute the size of each size level?

4. If every free path of a graph $G$ with size $n$ is contained in a cycle, is $\mathcal{Q}_{n}(G)$ hamiltonian? 
5. Is every size level of a hamiltonian graph hamiltonian?

It is well known that the 1-st size level is hamiltonian for hamiltonian graphs (Theorem 4.33 in [1])

6. Given a graph $G$ with size $N$, is $\mathcal{Q}_{N-1}(G)$ hamiltonian?

7. If $\mathcal{Q}_{n}(G)$ is hamiltonian, is $\mathcal{Q}_{n+1}(G)$ hamiltonian?

8. What properties are size properties?

9. What properties are size reversible properties?

10. What properties are such that, if a size level has the property, then the original graph has that property?

\subsection{QUESTIONS ABOUT $\mathcal{C}(\mathrm{G})$}

1. We showed a characterization of graphs $G$ which $\mathcal{C}(G)$ can be embedded in the plane. Study embeddings of $\mathcal{C}(G)$ to other surfaces.

2. Given a connected graph, can we find a formula to compute the number of vertices of $\mathcal{C}(G) ?$

3. Given a connected graph, can we find a formula to compute the size of $\mathcal{C}(G)$ ? 
4. For which graphs $G$ is the graph $\mathcal{C}(G)$ hamiltonian?

Note that paths and cycles are these kind of graphs (Propositions 119 and 120). The 3-star is not (Theorem 123).

5. Is the induced set $\mathcal{P}\left(G_{X}\right)$ homeomorphic to $C(X)$ ?

\subsection{OTHER HYPERSPACES}

As we mention in Chapter 1, given a topological space, a hyperspace of that space is a specific collection of subsets with a special property. In Chapters 1 and 5 we mention the hyperspace $C(X)$. Aside from $C(X)$, which we discussed in Chapters 1 and 5 , there are other hyperspaces. Given a continuum $X$, here are examples of collection of subsets, that together with the topology induced by the metric in Definition 153, they are hyperspaces of $X$ :

$$
\begin{gathered}
2^{X}=\{A \subset X: A \text { is nonempty and closed }\} \\
C_{n}(X)=\left\{A \in 2^{X}: A \text { has at most } n \text { components }\right\} \\
F_{n}(X)=\left\{A \in 2^{X}: A \text { has at most } n \text { elements }\right\}
\end{gathered}
$$

Note that $C(X), C_{n}(X)$ and $F_{n}(X)$ are all subspaces of $2^{X}$. See [5] for more information about those hyperspaces.

The natural problem is :

1. Define the hyperspace graphs in abstract graph theory that are analogous to the topological hyperspaces $2^{X}, C_{n}(X)$ and $F_{n}(X)$. 
In other words, given a positive integer $n$ and, the vertex sets:

$$
\begin{gathered}
\{K \subset G: K \text { is a subgraph of } G\} ; \\
\{K \subset G: K \text { has } n \text { components }\} \\
\{K \subset G: K \text { has at most } n \text { vertices and no edges }\}
\end{gathered}
$$

2. Define an adjacency relation in those sets compatible with the adjacency given for $\mathcal{C}(G)$

3. Which of those hyperspace graphs are such that two graphs having isomorphic hyperspace graphs implies that the graphs are isomorphic?

4. Is there a relation between some geometric realization of those hyperspace graphs and their topological analogues?

\subsection{INFINITE GRAPHS AND DIGRAPHS}

In abstract graph theory, a graph can be generalized by allowing the vertex set to be infinite. These are called infinite graphs.

1. Define hyperspace graphs for infinite graphs.

A digraph is defined just like a graph, except that the edges are ordered pairs of vertices.

2. Define hyperspace graphs for digraphs. 


\section{Bibliography}

[1] G. Chartrand and L. Lesniak, Graphs $\&$ Digraphs, 4th Ed, CRC Press (2004).

[2] R. DudA, On the Hyperspace of Subcontinua of a Finite Graph, I, Fund. Math. 62 (1968), 265-286.

[3] R. DudA, On the Hyperspace of Subcontinua of a Finite Graph, II, Fund. Math. 63 (1968), 225-255.

[4] J. L. Gross and J. Yellen, Handbook of Graph Theory, Discrete Mathematics and Its Applications, CRC Press (2004)

[5] A. Illanes and S. B. Nadler, JR., Hyperspaces, Fundamentals and Recent Advances, Monographs and Textbooks in Pure and Applied Math., Vol. 216, Marcel Dekker Inc., New York, N.Y., 1999.

[6] K. Kuratowski, Sur le probléme des courbes gauches en topologie, Fund. Math. 15 (1930), 271-283.

[7] S. B. Nadler, JR., Hyperspaces of Sets, Monographs and Textbooks in Pure and Applied Math., Vol. 49, Marcel Dekker Inc., New York, N.Y., 1978. 
[8] S. B. Nadler, JR., The Hyperspace Graph, Pre-print. 
803 ALPINE ST. • APARTMENT 7 • MORGANTOWN, WV 26505-2662

MOBILE(304)826-6290・E-MAIL LIKIN@MATH.WVU.EDU

\section{LIKIN C.SIMON ROMERO}

DATE OF BIRTH: December 12 th 1974

BIRTH PLACE: Mexico City, Mexico.

NATIONALITY: Mexican

1992 - $2000 \quad$ Universidad Nacional Autonoma de Mexico $\quad$ Mexico City, Mex.

Bachelors degree in Mathematics

- Advisor: Dr. Alejandro Illanes Mejia.

2000 - 2005 West Virginia University

Morgantown, WV

Ph. D. degree in Mathematics

- Advisor: Dr. Sam B. Nadler, Jr.

- Date of Defense: May 27th 2005

Spanish and English.

Dendrites with a closed set of endpoints. Topology and its Applications 115 (2001), pp. 1-17.

Means in Continua (Spanish). Miscélanea Matemática 33 (2001), pp. 99-98.

Means with special properties. Houston Journal of Mathematics 29 No.2 (2003), pp. 313-324.

AWARDS AND SCHOLARCHIPS RECEIVED

PAPIIT Scholarship from UNAM (1998).

Internship in the Institute of Mathematics, UNAM (1998-2000)

Sotero Prieto Outstanding Thesis Award, UNAM (2000)

Summer Research Fellowship, West Virginia University (2003) 
HERF Supplemental Scholarship, West Virginia University (2004).

Outstanding Graduate Teaching Assistant in Mathematics, West Virginia University (2005)

\section{PROFESSIONAL EXPERIENCE}

1998-1999 Colegio Madrid Mexico City, Mex.

High School Teacher

- Course: Introduction to Calculus

1998-2000 Universidad Nacional Autonoma de Mexico Mexico City, Mex. Teacher Assistant

- Courses: Advanced Topics in Geometry I and II, Mathematics Teaching Seminar II, Projective Geometry.

2000-present West Virginia University Morgantown, WV

Graduate Teacher Assistant

- Courses: Calculus I, Calculus II, Multivariable Calculus, College Algebra and Applications and Pre-Calculus for Business.

ADDITIONAL PROFESSIONAL ACTIVITIES

Tutoring students of various levels.

Human Resources Advisor for Publishing Company in Mexico.

Advisor for the Self-Learning Program of the CONAFE (National Council for the Promotion of Education) in Mexico.

Participant of the Literacy Campaign organized by the Colegio Madrid in two years (1991 and 1992).

Advisor of the Literacy Campaign Program in the Colegio Madrid (19981999)

\section{TALKS AT CONFERENCES AND SEMINARS}

Summer 1997 Continuum Theory Seminar Mathematics Institute, UNAM

Summer 1997 2nd Meeting “Two Days in Continuum Theory” $\quad$ BUAP, Mexico

Fall 1999 32 nd National Meeting of the Mexican Mathematics Society.

Winter 1999 Intern Seminar Mathematics Institute, UNAM 
Fall 2000 Continum Theory Seminar West Virginia University

Summer $2001 \quad 1^{\text {st }}$ Continuum Theory Mini-Conference Delaware University

Spring 2002 Graduate Seminar West Virginia University

Summer $20021^{\text {st }}$ International Meeting in Continuum Theory $\quad$ BUAP, Mexico

Fall 2002 Combinatorics Seminar West Virginia University

Fall $2003 \quad 2^{\text {nd }}$ Continuum Theory Mini-Conference West Virginia University

Summer $20049^{\text {th }}$ Chico Topology Conference California State University, Chico

Fall 2004 Topology Seminar Pittsburgh University

Spring 2005 Combinatorics Seminar West Virginia University

Spring 2005 Topology Seminar West Virginia University

Spring 2005 Spring Topology and Dynamics Conference Berry College

TRAINING

Attended the Teaching Seminar at West Virginia University.

PROFESSIONAL MEMBERSHIPS

Current Member of the AMS 